\title{
Non-perturbative computation of the strong coupling constant on the lattice
}

\author{
Rainer Sommer ${ }^{\mathrm{a}, \mathrm{b}}$, Ulli Wolff ${ }^{\mathrm{b}, *}$ \\ ${ }^{a}$ John von Neumann Institute for Computing (NIC), DESY, Platanenallee 6, 15738 Zeuthen, Germany \\ ${ }^{b}$ Institut für Physik, Humboldt Universität, Newtonstr. 15, 12489 Berlin, Germany
}

\begin{abstract}
We review the long term project of the ALPHA collaboration to compute in QCD the running coupling constant and quark masses at high energy scales in terms of low energy hadronic quantities. The adapted techniques required to numerically carry out the required multiscale non-perturbative calculation with our special emphasis on the control of systematic errors are summarized. The complete results in the two dynamical flavor approximation are reviewed and an outlook is given on the ongoing three flavor extension of the programme with improved target precision.
\end{abstract}

Keywords: QCD, running coupling, quark mass, lattice QCD, Monte Carlo, Schrödinger functional, gradient flow

\section{Introduction}

Quantum Chromo Dynamics (QCD) is the renormalizable quantum field theory containing gluon and quark fields that interact in a unique way dictated by SU(3) gauge invariance. It may be seen as arising from the standard model of elementary particles in a limit where all other fields, including their interactions with quarks and gluons, are stripped away. Strong interactions and confinement are the characteristics of this sector which hence calls for non-perturbative evaluations and is in the focus of lattice formulations and simulations.

We here consider QCD with a free number of $N_{\mathrm{f}}$ color triplets (flavors) of quark species. In Nature we see the case $N_{\mathrm{f}}=6$ with the flavors up, down, strange, charm, bottom and top in order of ascending mass. The species beyond light up and down quarks come with characteristic scales of the order of $0.1 \mathrm{GeV}, 1 \mathrm{GeV}, 4 \mathrm{GeV}$, $175 \mathrm{GeV}$. Therefore it makes sense to consider effective theories with $N_{\mathrm{f}}<6$ to describe physics with characteristic energies significantly below the scales of the dropped degrees of freedom. They then enter only indirectly into the determination of the free parameters of

\footnotetext{
${ }^{*}$ Corresponding author

Email address: uwolff@physik.hu-berlin.de (Ulli Wolff)
}

the effective theory. In lattice simulations the modelling of the precise flavor content is technically very demanding. Therefore a lot of studies are found and will also be discussed here that refer to $N_{\mathrm{f}}=2$ and $N_{\mathrm{f}}=3$ where the latter number is the minimum to allow for real applications as an effective theory $[1,2,3,4,5]$. In any case generalized QCD has $N_{\mathrm{f}}+1$ free parameters given by one quark mass per species and in addition the gauge coupling.

The two light species are in most studies, including those described here, approximated to be degenerate. The value zero for some or even all $N_{\mathrm{f}}$ quark masses is theoretically nice as it enhances the chiral symmetry of the model and is thus stabilized under renormalization. The renormalization of the coupling can be defined in this massless limit and we then speak of a massless renormalization scheme. Such schemes are technically convenient in nontrivial perturbative as well as non-perturbative calculations. The renormalization of the coupling can be left unchanged as quark masses are 'turned on later'. To define a renormalized coupling constant in a massless scheme an additional scale $\mu$ enters via the renormalization conditions. The resulting scale dependent 'running' coupling $\bar{g}(\mu)$ obeys a Callan- 
Symanzik equation

$$
\mu \frac{d}{d \mu} \bar{g}(\mu)=\beta(\bar{g}(\mu))
$$

in which the function $\beta$ is determined by the theory once a particular coupling definition has been adopted. A negative $\beta$-function corresponds to asymptotic freedom. The free integration constant that arises in solving this differential equation can be taken as the free parameter that the bare coupling has been 'traded for' in the process of renormalization. It may be fixed by specifying $\bar{g}$ for a specific $\mu$ value in $\mathrm{GeV}$. Alternatively one may convert the Callan-Symanzik equation into the equivalent integral statement that

$$
\begin{aligned}
\Lambda & =\mu\left(b_{0} \bar{g}^{2}(\mu)\right)^{-b_{1} / 2 b_{0}^{2}} \exp \left[-1 /\left(2 b_{0} \bar{g}^{2}(\mu)\right)\right] \\
& \times \exp \left[-\int_{0}^{\bar{g}(\mu)}\left\{\frac{1}{\beta(x)}+\frac{1}{b_{0} x^{3}}-\frac{b_{1}}{b_{0}^{2} x}\right\} d x\right]
\end{aligned}
$$

is independent of $\mu$. In this equation $b_{0}, b_{1}$ are the leading and scheme independent coefficients in the asymptotic expansion

$$
\begin{aligned}
& \beta(x)=-\sum_{n \geq 0} b_{n} x^{2 n+3}, \\
& b_{0}=\frac{1}{(4 \pi)^{2}}\left(11-\frac{2}{3} N_{\mathrm{f}}\right), \\
& b_{1}=\frac{1}{(4 \pi)^{4}}\left(102-\frac{38}{3} N_{\mathrm{f}}\right) .
\end{aligned}
$$

For asymptotically large $\mu$ it is sufficient to evaluate (2) with the perturbative series for $\beta$ truncated beyond some $n \geq 1$. Therefore, in a perturbative context, $\Lambda$ is associated with the behavior of $\bar{g}(\mu)$ for $\mu \rightarrow \infty$.

\section{Hadronic renormalization scheme and finite size scaling}

In lattice simulations also non-perturbative quantities associated with scales of order one $\mathrm{GeV}$ and below can be computed in principle. Examples are the masses of light hadrons and matrix elements involving their oneparticle states, decay constants like $f_{\pi}, f_{\mathrm{K}}$ for example $[6,7]$. This opens up the possibility to also match such quantities directly to experiment and in this way determine the free parameters of QCD which can then be determined with an in principle arbitrary precision ${ }^{1}$. This

\footnotetext{
${ }^{1}$ This refers to pure QCD. Other interactions are still neglected.
}

is not true if perturbation theory at any finite energy is involved, since with an asymptotic expansion - even if very high orders were available - an uncertainty remains. This effect is expected to be small at the Z-mass, but the situation is much more delicate for example for determinations of $\alpha_{s}$ in the $\tau$-mass region.

As a conceptually simple example of a hadronic scheme one could imagine to use as input parameters the mass of the proton and in addition the masses of $N_{\mathrm{f}}$ types of stable mesons that are sensitive to the respective quark masses. In practice one of course has $N_{\mathrm{f}}+1$ dimensionless parameters at ones disposal in the lattice theory of which $N_{\mathrm{f}}$ may be determined by dialing the correct ratios of meson to proton mass. The remaining degree of freedom allows to tune the lattice theory to its critical point where the continuum limit is reached. Due to asymptotic freedom in QCD this is accomplished by sending the bare coupling to zero. In this limit, all dimensionfull quantities emerge in the form of well-defined multiples of appropriate powers of the proton mass which we thus employ to set the scale for all observables. Equivalently we may say that all that is computed from theories including the lattice and compared with experiment are dimensionless ratios of observables. The above scheme selects a minimal set of independent mass ratios and, with these tuned, all other ratios must 'fall in place'. We try to be very explicit on this seemingly trivial issue, as sometimes confusion seems to arise here nevertheless.

In the previous paragraphs we have described a rather idealized situation. For various technical reasons we will not use this precise hadronic scheme, and in addition several sources of in practice unavoidable systematic errors have to be taken into account in lattice computations.

A lattice that is simulated on a computer necessarily has a finite number of sites and thus finitely many degrees of freedom. This implies a finite extent $L$ and a finite spacing or resolution $a$ such that one has $(L / a)^{4}$ sites. In large present day simulations $L / a \sim 100$ is achieved. If we refer to $m_{\text {had }}$ as some hadronic mass scale, then $a m_{\text {had }}>0$ represents a distortion of the physics by an unphysical UV cutoff effect. Details depend on the chosen lattice discretization, but in practice and employing Symanzik's theory of cutoff effects $[8,9,10]$, we expect these effects to diminish asymptotically at a rate proportional to $\left(a m_{\mathrm{had}}\right)^{2}$. We need to verify that we have reached this asymptotic behavior to estimate the prefactor by multiple simulations in which the resolution (and nothing else) is varied. This whole procedure is called continuum extrapolation and, of course, leaves behind a contribution in the final error 
budget.

In the same way, unless finite size effects are deliberately looked for (see below), also a finite product $L m_{\text {had }}<\infty$ is an unwanted IR cutoff effect to be extrapolated away or at least bounded. The situation here is however more benign, as theory [11] implies that the infinite volume limit is reached at an exponential rate proportional to $\exp \left(-m_{\pi} L\right)$, where the pion with mass $m_{\pi}$ enters as the lightest degree of freedom. In todays large volume lattice simulations we are largely restricted to cutoffs $L \leq 6 \mathrm{fm}$ and $a^{-1} \leq 5 \mathrm{GeV}$, although these extreme values cannot yet quite be realized simultaneously and compromises, depending on the physics studied, have to be made.

One would clearly like to confront results extracted by matching perturbation theory to experiment at high enough energy with those of a hadronic scheme. This amounts to nothing less than establishing QCD as one theory at all these length scales. An obvious strategy is to compute the renormalized parameters of a perturbative scheme from 'within' a hadronic scheme. Focussing on the coupling constant this would require to compute beside a hadron mass $m_{\text {had }}$ some scale dependent observable $O(\mu)$ that possesses a perturbative expansion

$$
O(\mu)=\alpha(\mu)+p_{1} \alpha^{2}(\mu)+p_{2} \alpha^{3}(\mu)+\ldots
$$

where the coupling $\alpha$ refers to some perturbative scheme like $\overline{\mathrm{MS}}$. This evaluation has to proceed at a high enough scale $\mu / m_{\text {had }}=\rho \gg 1$ to get sufficient perturbative precision in extracting $\alpha$ at energy $\rho \times m_{\text {had }}$. With the help of (2) this information may be converted to a value for $\Lambda / m_{\text {had }}$. As is well known, the $\Lambda$ parameter of any other scheme follows now by relating the corresponding couplings at one loop order.

If we imagine to perform such a calculation naively on the lattice, we have to cope with a multiscale problem where we have to satisfy the string of inequalities

$$
a \ll \mu^{-1} \ll m_{\text {had }}^{-1} \ll L .
$$

Given the practical constraints on $L / a$ it is clear that such a direct approach will require severe compromises. An overview over various approaches including the direct one is found in [6].

Due to these difficulties strategies have been devised to alleviate the problem by circumventing one of the required large scale ratios. One idea is to try to tolerate the scales $a^{-1}$ and $\mu$ to lie in the same range and thus perform perturbation theory at the scale of the cutoff. Such a calculation, as well as references to earlier versions, is discussed in [12]. A valid criticism in our opinion is, that it thus becomes hard to disentangle UV cutoff effects from limitations of (truncated) perturbation theory. To control lattice artefacts it seems necessary to be able to vary the lattice spacing over some range with physical scales $m_{\text {had }}, \mu$ held fixed.

Our finite size strategy which is in the focus of the remainder of this article may be seen as identifying $\mu$ and $L^{-1}$ over a major part of the calculation. We will exploit the fact that finite size effects are universal predictions of quantum field theory. The Casimir force in QED [13] is such a finite size effect which has been experimentally confirmed as a subtle manifestation of vacuum fluctuations. We extend this concept to more abstract cases involving periodic or Dirichlet type boundary conditions on finite systems. They are not realized in the laboratory, but universality here means that there again are unique predictions for effects depending on the size $L$, independent of how the field theory is regularized and the UV cutoff limit in the finite box is taken.

We now sketch such a computation and come back to details in the later sections. The key quantity required in our approach is an $L$-dependent finite size observable $\bar{g}(L)$ that can function as a non-perturbatively defined coupling constant. It must exist for arbitrary $L$ and possess a manageable perturbative expansion that is applicable at small size, for $L^{-1}$ much larger than hadronic scales. With a suitable normalization $\bar{g}(L)$ will be related to other couplings like for example

$$
\bar{g}^{2}(L)=\bar{g}_{\overline{\mathrm{MS}}}^{2}(\mu)+c(\mu L) \bar{g}_{\overline{\mathrm{MS}}}^{4}(\mu)+\mathrm{O}\left(\bar{g}_{\overline{\mathrm{MS}}}^{6}\right)
$$

where for good perturbative accuracy one will take $\mu L=\mathrm{O}(1)$. Beside perturbation theory $\bar{g}(L)$ must be easily computable numerically and a reasonable signal to noise ratio for its estimator is another practical requirement. Appropriate such couplings are known for Schrödinger functional boundary conditions, see the later sections for details.

With $\bar{g}(L)$ at hand we now first consider a simulation in a large, i. e. effectively infinite, volume where we tune the bare parameters to achieve $a \ll m_{\text {had }}^{-1} \ll$ $L$. A hadronic scale $m_{\text {had }}$, or rather the dimensionless combination $a m_{\text {had }}$ is the output. Then, for the same bare parameters we diminish $L / a$ to the point where $L m_{\text {had }} \equiv(L / a)\left(a m_{\text {had }}\right)=\rho$ becomes a fixed number of order unity (the previous value $a m_{\text {had }}$ is used). At this size $\bar{g}(L)$ will not be small but its value can be computed by simulation. By repeating these steps for several small $a m_{\text {had }}$ and extrapolating to the continuum we thus derive a numerical value $\bar{g}(L)$ at the scale $L=\rho m_{\text {had }}^{-1}$ in the non-perturbative regime. This is a universal number $\bar{g}\left(L_{\max }=\rho m_{\text {had }}^{-1}\right)$ in the continuum where $L_{\max }$ can be cited in fermi once $m_{\text {had }}$ has been related to experiment. 
What remains to be done now is to evolve $\bar{g}(L)$ from the now known starting point at $L_{\max }$ to other $L$ small enough to make contact with couplings like $\bar{g}_{\overline{\mathrm{MS}}}$ by perturbation theory. The Callan Symanzik equation is a differential description of such an evolution guided by the corresponding $\beta$-function. For a non-perturbative evolution the change of scale by a finite factor two seems more natural. This leads to the definition of a finite stepsize counterpart of a $\beta$-function, the step scaling function [14]

$$
\sigma(u)=\left.\bar{g}^{2}(2 L)\right|_{\bar{g}^{2}(L)=u} .
$$

Note that $\sigma$ does not refer to the lattice at this point but is a universal continuum quantity, different however for differently defined couplings. Before we outline the computation of the function $\sigma$ we discuss its application. We use it to build a sequence $\left\{u_{i}, i=0,1, \ldots, n\right\}$ based on the recursion $u_{i}=\sigma\left(u_{i+1}\right)$ and started at $u_{0}=\bar{g}^{2}\left(L_{\max }\right)$. This immediately implies that

$$
u_{n}=\bar{g}^{2}\left(2^{-n} L_{\max }\right)
$$

holds and that for sufficiently many steps this value is arbitrarily deep in the perturbative regime. Here in addition we should also start to see an evolution that coincides with the one produced by the Callan Symanzik equation with a perturbative approximation for the appropriate $\beta$ function. In figure 1 a schematic view is given for the example of $\bar{g}$ realized by the Schrödinger functional finite volume coupling that will be detailed below.

$$
\begin{array}{ccc}
L_{\text {max }}=\mathrm{O}\left(\frac{1}{2} \mathrm{fm}\right): \quad \mathrm{HS} \longrightarrow \quad \mathrm{SF}\left(\mu=1 / L_{\text {max }}\right) \\
\downarrow \\
\mathrm{SF}\left(\mu=2 / L_{\text {max }}\right) \\
\downarrow \\
\bullet \\
\\
\\
& & \bullet \\
& \mathrm{SF}\left(\mu=2^{n} / L_{\text {max }}\right) \\
& \mathrm{PT}: \quad \downarrow \\
\text { DIS, jet-physics, at } s=M_{\mathrm{Z}}^{2} \quad \stackrel{\mathrm{PT}}{\longleftarrow} \quad \Lambda_{\mathrm{QCD}}
\end{array}
$$

Figure 1: The strategy for a non-perturbative computation of short distance parameters. SF refers to the Schrödinger functional renormalization scheme and HS to a hadronic scheme.

It remains to outline the computation of $\sigma$. To this end we pick a resolution $L / a$ and some value $u$ and tune

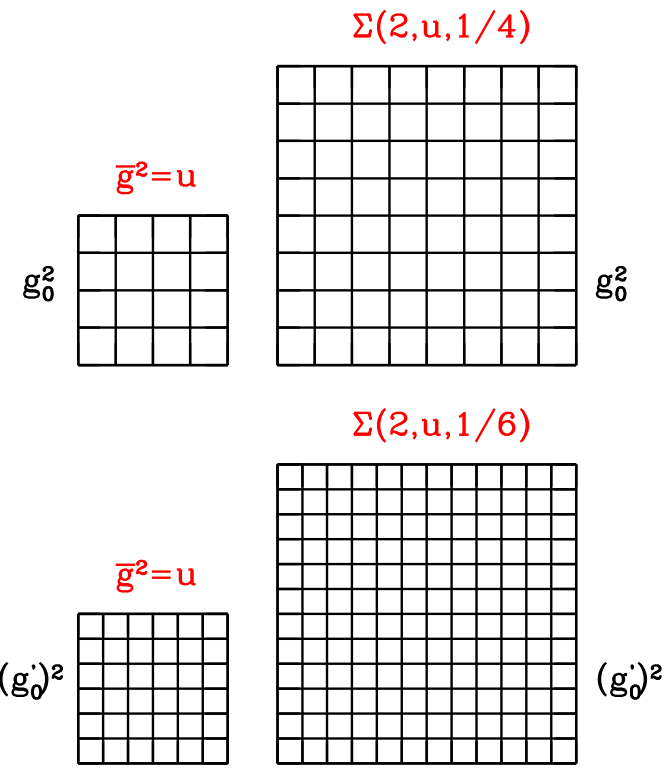

Figure 2: Illustration of the computation of the continuum step scaling function from finer and finer lattices. Note that $\Sigma(2, u, a / L)$ in the illustration corresponds to our lattice step scaling function $\Sigma(u, a / L)$.

the bare parameters such that $\bar{g}^{2}=u$ results and that the quark masses vanish (massless scheme). Then, keeping the bare parameters fixed, we double the size to $2 L / a$ and determine a value of the lattice step scaling function $\Sigma(u, a / L)=\bar{g}^{2}(2 L)$. If this procedure is repeated for a sequence of $L / a$ we finally extrapolate to

$$
\sigma(u)=\lim _{a / L \rightarrow 0} \Sigma(u, a / L)
$$

which again is a piece of universal continuum physics. We need to implement this at a sufficiently dense set of $u$ values to have a sufficiently precise control over $\sigma(u)$ in the range required for the evolution (10). The procedure is indicated in figure 2 for one value of $u$.

\section{Lattice discretization}

\subsection{The action}

The gluon vector potential $A_{\mu}(x)$ in Euclidean continuum QCD has its values in the Lie algebra SU(3) and enters as a parallel transporter over infinitesimal distances into the covariant derivative $D_{\mu}=\partial_{\mu}+A_{\mu}$. The resulting gauge covariant curvature $F_{\mu \nu}=\left[D_{\mu}, D_{\nu}\right]$, also in the Lie algebra, is the building block of the gauge invariant action density that is integrated over in the non- 
negative $^{2}$ Euclidean Yang-Mills action

$$
S_{\text {cont }}=-\frac{1}{2 g_{0}^{2}} \int d^{4} x \operatorname{tr}\left(F_{\mu \nu} F_{\mu \nu}\right) .
$$

Following Wilson [15] we put strong emphasis on gauge invariance and construct the discretization on a hypercubic lattice in a way that is manifestly compatible with this structure. The smallest separation on the lattice are finite links $(x, \mu)$ starting from any site $x$ into the $\mu$-direction and ending at its nearest neighbor $x+a \hat{\mu}$ ( $a$ is the lattice spacing and $\hat{\mu}$ a unit vector). The associated gauge transporters $U(x, \mu)$ are $\mathrm{SU}(3)$ group-valued and represent the fundamental gluon field on the lattice. In the path integral they are integrated over with the invariant Haar measure independently on each link. Curvature on the lattice shows up by the two-step transporters $U(x, \mu) U(x+a \hat{\mu}, v)$ and $U(x, v) U(x+a \hat{v}, \mu)$ being different or, equivalently, by the plaquette field

$$
\begin{aligned}
& P(x, \mu, v)= \\
& U(x, \mu) U(x+a \hat{\mu}, v)[U(x, v) U(x+a \hat{v}, \mu)]^{-1}
\end{aligned}
$$

differing from unity. Thus a direct transcription of (12) onto the lattice is given by the Wilson plaquette action

$$
S_{\mathrm{W}}=\beta \sum_{x, \mu<v} \operatorname{Re} \operatorname{tr}[1-P(x, \mu, v)] .
$$

If we build $U(x, \mu)$ out of slowly varying infinitesimal continuum $A_{\mu}(x)$ we find that $S_{\mathrm{W}}$ approaches $S_{\text {cont }}$ with $\beta=6 / g_{0}^{2}$, i. e. (14) is a classically valid discretization.

In the same reference [15] Wilson has also introduced what today are called Wilson lattice fermions, which will be the discretization of the quark Dirac field employed here. Independent quark fields $\psi(x)$ are introduced on the sites of the lattice. They carry a fourvalued Dirac index, the SU(3) color index and an $N_{\mathrm{f}}$ valued flavor index which we all suppress in our notation. The covariant forward and backward derivatives of quarks are defined by

$$
\begin{aligned}
& \left(D_{\mu} \psi\right)(x)=\frac{1}{a}[U(x, \mu) \psi(x+a \hat{\mu})-\psi(x)] \\
& \left(D_{\mu}^{*} \psi\right)(x)=\frac{1}{a}\left[\psi(x)-U^{-1}(x-a \hat{\mu}, \mu) \psi(x-a \hat{\mu})\right]
\end{aligned}
$$

It is well known that the most obvious discretized Dirac operator leads to spurious particle poles, the so-called fermion doubling problem. Wilson's remedy for this problem is the addition of a term proportional to a discretized form of the covariant Laplacian, the Wilson

\footnotetext{
${ }^{2}$ In our convention $A_{\mu}$ and $F_{\mu \nu}$ are antihermitean.
}

term, which attributes a mass of order $a^{-1}$ to the doubler modes but is otherwise suppressed by an extra power of $a$. The total Wilson Dirac operator is now given by

$$
D_{\mathrm{W}}=\frac{1}{2} \gamma_{\mu}\left(D_{\mu}+D_{\mu}^{*}\right)-\frac{a}{2} D_{\mu} D_{\mu}^{*}
$$

The total lattice QCD action is thus given by

$$
S_{\mathrm{W} ; \mathrm{W}}[U, \psi, \bar{\psi}]=S_{\mathrm{W}}[U]-\sum_{x} \bar{\psi}\left(D_{\mathrm{W}}+M_{0}\right) \psi,
$$

where $M_{0}$ is the bare mass matrix with the mass parameters for the various flavors on its diagonal. All these ingredients may now be finally assembled to write down the lattice QCD partition function

$$
Z=\int D U D \psi D \bar{\psi} \exp \left\{-S_{\mathrm{W} ; \mathrm{w}}[U, \psi, \bar{\psi}]\right\}
$$

which beside the group integrations requires additional integrations over the Grassmann valued quark fields. For numerical simulations the latter (Gaussian) integrals are carried out and produce the fermion determinant

$$
Z=\int D U \exp \left\{-S_{\mathrm{W}}[U]\right\} \operatorname{det}\left(D_{\mathrm{W}}+M_{0}\right) .
$$

The matrix $D_{\mathrm{W}}$ has $U$ fields in its matrix elements and the determinant represents a complicated nonlocal effective action in $U$ that has to be taken into account for sampling $U$ with the weight given by this integrand. The standard hybrid Monte Carlo algorithms are able to cope with this, but nevertheless here is the source of the enhanced complexity of simulations once dynamical quark degrees of freedom are included. Some more details on our algorithmic implementation of the QCD path integral will be given in section 6 .

Another important point to mention is that the Wilson fermion regularization breaks chiral symmetry which emerges only in the continuum limit. Due to the Wilson term, $D_{\mathrm{W}}$ does not anticommute with $\gamma_{5}$. As a consequence the masses on the diagonal of $M_{0}$ undergo additive renormalization and the physical zero mass condition to set up a massless scheme has to be enforced as a nontrivial renormalization condition - usually some chiral Ward identity - which will force $a M_{0}$ to approach a $g_{0}$ dependent nontrivial critical value $a m_{c}\left(g_{0}\right)$. Note that in perturbation theory one finds $a_{c}\left(g_{0}\right)=c_{1} g_{0}+\mathrm{O}\left(g_{0}^{2}\right)$ which amounts to a linearly diverging bare mass parameter

\subsection{Symanzik improved action}

A further consequence of missing chiral symmetry in the regularized theory is the appearance of cutoff effects that are linear in the lattice spacing $a$ (multiplied 
by powers of logarithms). In Symanzik's approach the structure of cutoff effects can be studied by describing the lattice theory including leading cutoff effects by an effective theory in the continuum. This requires additional terms in the action of the latter beyond the combination of renormalizable terms of dimension up to four that we have discretized before. In asymptotically free theories the dimension of terms can be used to organize the additional contributions to the action: extra terms of dimension five represent lattice artefacts that vanish linearly in $a$, dimension six those that are quadratic and so on. The second essential criterion is to only admit terms that are invariant under the (reduced) symmetry that is still present in the lattice theory. Here chiral symmetry is missing for Wilson fermions. The interplay between dimension and symmetry leads to a finite number of additional couplings that allows to match all cutoff effects up to a given order in $a$. This is the standard situation in effective theories, with a rapid proliferation of the number of terms as the order is increased. In addition, all that one can hope for is an asymptotic expansion that is relevant close to the continuum limit where combinations like $a m_{\text {had }}$ are already small. In addition to the enlarged action also in renormalized observables extra mixings with higher dimensional terms of the same symmetry have to be taken into account to achieve a complete representation of cutoff effects in correlation functions. The whole concept may be seen as an extension of the renormalization programme that normally just focuses on divergences ( $\log a$ and possibly inverse powers) to small positive powers of $a$.

The dimension five terms relevant for the linear order in $a$ have been classified and listed in [16] for mass degenerate quarks. In this case there are five operators of which three can be absorbed into modifications of the bare coupling and the bare mass. The remaining two operators are the Pauli term

$$
O_{1}=\bar{\psi} \sigma_{\mu \nu} F_{\mu \nu} \psi
$$

and

$$
O_{2}=\bar{\psi} D_{\mu} D_{\mu} \psi+\bar{\psi} \overleftarrow{D}_{\mu} \overleftarrow{D}_{\mu} \psi
$$

Note that within the Symanzik effective theory we systematically expand in $a$. Therefore the dimension five terms in the action with an explicit factor $a$ are expanded down from the exponent and appear as operator insertions.

The description and ultimately elimination of a class of cutoff effects becomes much more manageable if we restrict ourselves to what is called on-shell improvement [10]. The restriction refers to correlations with a finite number of local operators which all reside at physical separations from each other, finite multiples of $m_{\text {had }}^{-1}$ for example. Then we can deform the integration variables of the path integral at all points without inserted operators to derive the so-called equations of motion as operator identities. They can be used to transform between the higher dimension terms classified before and to reduce their number while still matching cutoff effects in on-shell correlations. For the case at hand the equation of motion is just the Euclidean Dirac equation

$$
\left(\gamma_{\mu} D_{\mu}+M_{0}\right) \psi=0, \quad \bar{\psi}\left(\gamma_{\mu} \overleftarrow{D}_{\mu}-M_{0}\right)=0
$$

where only the renormalizable terms are considered as we want to only modify the order five terms and neglect yet higher dimensional terms. This equation is usually employed to eliminate $\mathrm{O}_{2}$ and then the only new bulk term needed to describe $\mathrm{O}(a)$ on-shell cutoff effects in the effective theory is the Pauli term $O_{1}$. One more complication has to be mentioned. The insertions generated by the $a$-expanded improved action appear integrated over Euclidean space-time and thus are not strictly separated from the observables as the use of the equations of motion would require. A more detailed analysis confirms however that these violations of the equations of motion due to overlapping insertions ('contact terms') can be compensated in the observable improvement terms that were mentioned before.

The Symanzik description of cutoff effects can be used in a next step to eliminate these contributions by what is called Symanzik improvement. To that end a discretized version of the extra term(s) is added to the original action with coefficients that are tuned such that the corresponding couplings in the effective action vanish. This may then be seen as an alternative discretization without the leading artefacts that have been systematically canceled in this way. For the operator $O_{1}$ above this is the so called clover term first proposed in [17]. We then have the Sheikholeslami-Wohlert (SW) improved lattice action

$$
S_{\mathrm{W} ; \mathrm{SW}}=S_{\mathrm{W} ; \mathrm{W}}+c_{\mathrm{SW}} a^{5} \sum_{x} \bar{\psi}(x) \frac{i}{4} \sigma_{\mu \nu} \hat{F}_{\mu \nu}(x) \psi(x)(24)
$$

where the name clover is owed to the lattice representative of the field strength $\hat{F}_{\mu \nu}(x)$ from four co-planar plaquettes ('leafs')

$$
\begin{aligned}
& \hat{F}_{\mu \nu}(x)=\frac{1}{8 a^{2}}\left\{Q_{\mu \nu}(x)-Q_{\nu \mu}(x)\right\}, \\
& Q_{\mu \nu}(x)= P(x, \mu, v)+\text { three more } \\
& \text { rotated } 1 \times 1 \text { loops opened at } x,
\end{aligned}
$$


see figure 3. An improvement condition (generalized renormalization condition) that has to hold in the continuum theory has to be enforced to determine $c_{\mathrm{SW}}\left(g_{0}\right)$.

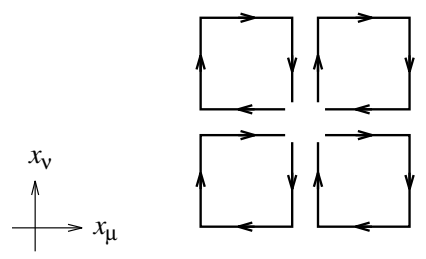

Figure 3: The clover leaf representation $\hat{F}_{\mu \nu}(x)$ of $F_{\mu \nu}(x)$.

The number of terms and coefficients required at leading order is small enough that we can and shall implement complete on-shell $\mathrm{O}(a)$ improvement. This is not practicable any more at the next order $a^{2}$. If one implements however only some part of the improvement terms with some prescription for the coefficients one still obtains a legal variant discretization different from the one without extra terms. Numerical experience suggests that the addition of a rectangle term to the gluon plaquette action with a strength suggested by improving at tree level of perturbation theory leads to a variant action with better properties than the pure plaquette form although artefacts are $\mathrm{O}\left(a^{2}\right)$ in both cases. This action, called tree level improved Lüscher-Weisz action, generalizes (14) and reads

$$
S_{\mathrm{LW}}=\beta \sum_{i=0}^{1} c_{i} \sum_{C \in \mathcal{S}_{i}} \operatorname{Re} \operatorname{tr}[1-P(C)] .
$$

Here $\mathcal{S}_{0}$ is the set of all different (unoriented) plaquette $(1 \times 1)$ loops on the lattice and $P(C)$ a parallel transporter around it. Hence, for $c_{0}=1, c_{1}=0$ this action would coincide with $S_{\mathrm{W}}$. The second term involves the set $\mathcal{S}_{1}$ of all different planar $1 \times 2$ loops (rectangles) and for the tree level improved Lüscher-Weisz action the weights assume the values

$$
c_{0}=\frac{5}{3}, \quad c_{1}=-\frac{1}{12} .
$$

\subsection{Improved currents and renormalization}

Quark currents are observables of primary importance in QCD. In particular the isovector axial current formed from the two light quarks

$$
A_{\mu}^{a}(x)=\bar{\psi} \gamma_{\mu} \gamma_{5} \tau^{a} \psi(x)
$$

and the pseudo-scalar density

$$
P^{a}(x)=\bar{\psi} \gamma_{5} \tau^{a} \psi(x)
$$

enter into the discussion of chiral symmetry. They are here given first as bare currents in terms of bare fields at the same lattice site and Pauli matrices $\tau^{a}$ operate on the up and down quarks. As discussed in the previous subsection for $\mathrm{O}(a)$ improvement these dimension three operators can mix with dimension four terms of the right symmetry. It turns out that there is no such term for $P^{a}$, but the improved axial current can mix with the gradient of $P^{a}$ and is hence given by

$$
\left(A_{\mathrm{I}}\right)_{\mu}^{a}(x)=A_{\mu}^{a}(x)+a c_{\mathrm{A}} \tilde{\partial}_{\mu} P^{a} .
$$

Here $\tilde{\partial}_{\mu}=\left(\partial_{\mu}+\partial_{\mu}^{*}\right) / 2$ is the symmetrized lattice derivative and $c_{\mathrm{A}}\left(g_{0}\right)$ is an improvement coefficient that has to be fixed by another improvement condition. It will turn out that its perturbative expansion starts at $\mathrm{O}\left(g_{0}^{2}\right)$.

In [16] the nontrivial interplay between the use of a massless renormalization scheme and improvement is discussed in some detail. In such a scheme all renormalization conditions are formulated at a normalization scale $\mu$. For nonzero, but for simplicity degenerate, quark masses $m_{0}$ the relation between bare and renormalized coupling must be taken as

$$
g_{\mathrm{R}}^{2}=\tilde{g}_{0}^{2} Z_{g}\left(\tilde{g}_{0}^{2}, a \mu\right), \quad \tilde{g}_{0}^{2}=g_{0}^{2}\left(1+b_{g} a m_{q}\right) .
$$

Here $b_{g}\left(g_{0}\right)$ is an improvement constant that eliminates $\mathrm{O}(a)$ effects at nonzero $m_{q}$ which in turn is the subtracted quark mass

$$
m_{q}=m_{0}-m_{c}\left(g_{0}\right)
$$

such that $m_{q}=0$ implies a vanishing physical mass. The term with $b_{g}$ reflects a dimension five term in the Symanzik effective action proportional to $m \operatorname{tr}\left(F_{\mu \nu}^{2}\right)$. It is only with this term (and the correct $b_{g}$ ) that in the process of expressing physical observables in terms of $g_{\mathrm{R}}$ not only divergences but also linear lattice artefacts are eliminated.

In a similar way the usual multiplicative mass renormalization must be replaced by

$$
m_{\mathrm{R}}=\tilde{m}_{q} Z_{m}\left(\tilde{g}_{0}^{2}, a \mu\right), \quad \tilde{m}_{q}=m_{q}\left(1+b_{m} a m_{q}\right) .
$$

Quite similar formulas follow for the current renormalizations

$$
\begin{aligned}
& \left(A_{\mathrm{R}}\right)_{\mu}^{a}=Z_{\mathrm{A}}\left(1+b_{\mathrm{A}} a m_{q}\right)\left(A_{\mathrm{I}}\right)_{\mu}^{a}, \\
& \left(P_{\mathrm{R}}\right)^{a}=Z_{\mathrm{P}}\left(1+b_{\mathrm{P}} a m_{q}\right) P^{a} .
\end{aligned}
$$

If in the continuum limit the chiral symmetry group $\mathrm{SU}\left(N_{\mathrm{f}}\right) \times \mathrm{SU}\left(N_{\mathrm{f}}\right)$ is recovered up to finite mass effects, then we expect the PCAC relation

$$
\partial_{\mu}\left(A_{\mathrm{R}}\right)_{\mu}^{a}=2 m_{\mathrm{R}}\left(P_{\mathrm{R}}\right)^{a}
$$




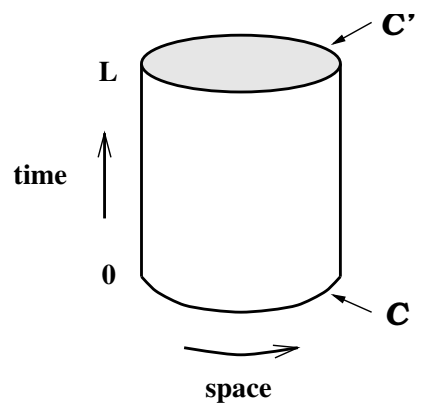

(LxLxL box with periodic b.c.)

Figure 4: The Schrödinger functional geometry.

to emerge as an operator relation that can be inserted into matrix elements. It is very advantageous to focus on this relation to actually define the renormalized mass in the improved theory, once the improved renormalized currents have been introduced already. The finite size Schrödinger functional scheme that we introduce next is a very convenient setting to do so.

Non-perturbative techniques to determine the improvement coefficients were developed in the quenched approximation $[18,19,20]$, and later applied for the two and three flavor theories in [21, 22, 23, 24]. Some coefficients such as $b_{\mathrm{P}}$ remain unknown non-perturbatively but can be taken from one-loop perturbation theory $[25,26]$.

\section{The Schrödinger functional}

For the Schrödinger functional (SF) [27] - and later the associated renormalization scheme - we consider a finite portion of Euclidean space time with spatial extent $L$ and temporal size $T$ as depicted in figure 4 . In the spatial directions $\mu=k=1,2,3$ we impose periodic boundary conditions $x \equiv x \pm L \hat{k}$ while Dirichlet boundary conditions fix certain field components at $x_{0}=0, T$ to externally given values. The SF can be defined in the continuum and is in fact studied by dimensionally regularized 1-loop perturbation theory in [27]. As we here want to mainly discuss non-perturbative computations we prefer to immediately start on the lattice, where some features of the SF even become simpler to discuss. This implies, of course, that both $L / a$ and $T / a$ must be integer.

\subsection{Gauge sector}

In the standard form of the SF the Dirichlet conditions for the gluon field $U(x, \mu)$ are

$$
\begin{aligned}
\left.U(x, k)\right|_{x_{0}=0} & =\exp \left(a C_{k}\right), \\
\left.U(x, k)\right|_{x_{0}=T} & =\exp \left(a C_{k}^{\prime}\right),
\end{aligned}
$$

where $C_{k}, C_{k}^{\prime}$ are constant Abelian vector potentials in the form of diagonal traceless imaginary matrices. Note that temporal links $U(x, 0)$ exist as integration variables for $x_{0}=0, a, \ldots, T-a$ and are not subject to any boundary conditions.

A possible interpretation of this Euclidean path integral with boundaries is in the Hamiltonian or transfer matrix formalism. It goes with a Schrödinger representation of states in Hilbert space as wave function(al)s on (three dimensional) spatial configurations $U(\mathbf{x}, k)$. We denote a state concentrated on a fixed field configuration $U(\mathbf{x}, k) \equiv \exp \left(a C_{k}\right)$ by a ket $|C\rangle$ (like $|x\rangle$-states in quantum mechanics). Then the SF partition function $\mathcal{Z}$ is equal to the matrix element

$$
\mathcal{Z}\left(C^{\prime} ; C\right)=\left\langle C^{\prime}\left|\mathrm{e}^{-T H} \mathbb{H}\right| C\right\rangle,
$$

where $\mathrm{e}^{-a \mathbb{H}}$ is the transfer matrix and $\mathbb{P}$ is a projector to gauge invariant states [28].

From the Euclidean point of view in the SF setup the time direction is distinguished from the others and there is no translation invariance in the time-direction. Therefore in this case we generalize the Wilson action to

$$
S_{\mathrm{Wsf}}=\beta \sum_{C \in \mathcal{S}_{0}} w(C) \operatorname{Retr}[1-P(C)] .
$$

Here the novelty is the plaquette dependent weight $w$ for which the transfer matrix formalism suggests to take $w(C)=1$ for all plaquettes except the purely spatial ones on the boundary where $w(C)=1 / 2$. This is so because it is natural to symmetrically distribute these contributions to the two adjacent transfer matrix factors (as for the potential $V(x)$ in quantum mechanics).

In the Symanzik effective action additional terms representing cutoff effects are possible due to the SF geometry. In the continuum they are given by three dimensional integrals over boundary planes with the dimension four densities $\operatorname{tr}\left(F_{0 k} F_{0 k}\right), \operatorname{tr}\left(F_{k l} F_{k l}\right)$ as integrands. These contributions are associated with $\mathrm{O}(a)$ boundary cutoff effects. For Symanzik improvement these terms are discretized and included in the lattice action with adjustable coefficients. For $S_{\mathrm{Wsf}}$ this is incorporated by two different nontrivial weights for space-time and for space-space plaquettes at the boundary. With our Abelian boundary fields the latter type does not contribute and we set

$$
w(C)=c_{t}\left(g_{0}\right)=1+c_{t}^{(1)} g_{0}^{2}+\ldots
$$

for $0 \mathrm{k}$ plaquettes touching the boundary and $w(C)=1$ for all others. In principle $c_{t}\left(g_{0}\right)$ has to be fixed by yet another improvement condition. In practice these terms can at present only be set to perturbative values 
as already indicated above. See [29] for a discussion of the relevance of this approximation. For the tree-level Lüscher Weisz action similarly modified weights are required for both plaquettes and rectangles close to the boundary which are discussed in [30] or more recently in [31]. In the following we always assume that these $\mathrm{O}(a)$ improvement terms are included in lattice actions for the SF.

To not introduce further scales the boundary fields $C, C^{\prime}$ are taken as multiples of $L^{-1}$ and an often used standard choice is

$$
\begin{aligned}
C_{k} & =\frac{i}{L} \operatorname{diag}(\eta-\pi / 3,-\eta / 2,-\eta / 2+\pi / 3) \\
C_{k}^{\prime} & =\frac{i}{L} \operatorname{diag}(-\eta-\pi, \eta / 2+\pi / 3, \eta / 2+2 \pi / 3) .
\end{aligned}
$$

The dimensionless parameter $\eta$ allows to vary the boundary values and is set to zero after taking derivatives with respect to it. In [27] it is shown that these particular boundary values (for not too small $L / a, T / a$ ) lead to a stable minimum of $S_{\mathrm{Wsf}}$. This minimum is unique up to gauge transformations which (at the boundaries) we restrict to the subgroup that preserves the boundary values. A representative $U(x, \mu)=\exp \left(a B_{\mu}\right)$ for this gauge orbit of minima is

$$
B_{0}=0, B_{k}=\left[x_{0} C_{k}^{\prime}+\left(T-x_{0}\right) C_{k}\right] / T
$$

which linearly interpolates between the boundaries.

We are now in a position to introduce the renormalized SF coupling. We start from the effective action or free energy

$$
\Gamma[B]=-\ln \mathcal{Z}\left(C^{\prime} ; C\right) .
$$

In perturbation theory a saddle point expansion around $B$ requires the usual Fadeev-Popov gauge fixing and yields a regular expansion

$$
\Gamma[B]=S_{\mathrm{Wsf}}[B]+\Gamma_{1}[B]+g_{0}^{2} \Gamma_{2}[B]+\ldots,
$$

where we note that the classical or tree level term $S_{\mathrm{Wsf}}[B]$ in (41) is proportional $g_{0}^{-2}=\beta / 6$. The definition of the coupling associated with the scale $L$ finally reads

$$
\bar{g}_{\mathrm{SF}}^{2}(L)=\left.g_{0}^{2} \frac{\partial S_{\mathrm{Wsf}} / \partial \eta}{\partial \Gamma / \partial \eta}\right|_{\eta=0} .
$$

In $\partial \Gamma / \partial \eta$ we differentiate the logarithm of a partition function with respect to a parameter entering into the (boundary terms of the) action. It immediately leads to an expectation value that is independent of unphysical factors in the path integral measure and can be estimated as a mean value of a well defined observable when field configurations are sampled with the probability $\exp \left(-S_{\mathrm{Wsf}}\right)$. The normalization factor $g_{0}^{2} \partial S_{\mathrm{Wsf}} / \partial \eta$ can easily be computed in closed form for (44).

\subsection{Quark sector}

The extension of the SF to fermions has been first presented in [32].

We generalize the periodic boundary conditions in space to a periodicity up to a phase $\mathrm{e}^{3}$ for the quark fields

$$
\psi(x+L \hat{k})=\mathrm{e}^{i \theta_{k}} \psi(x), \quad \bar{\psi}(x+L \hat{k})=\mathrm{e}^{-i \theta_{k}} \bar{\psi}(x),(48)
$$

which leaves all bilinear densities in the action and elsewhere strictly periodic. The angles $\theta_{k}$ allows us to vary the finite size kinematics in useful ways.

As for the Dirichlet boundary conditions in time, it turns out that only half of the components of the independent Grassmann fields $\psi$ and $\bar{\psi}$ have to be fixed. Formally this is a consequence of the first order nature of the Dirac equation and the correspondingly modified boundary value problem as was already noted in connection with the bag model [33]. Alternatively, and more laboriously, one may argue on the basis of the transfer matrix for Wilson fermions [34]. The result for the SF in any case are Dirichlet conditions

$$
\left.P_{+} \psi\right|_{x_{0}=0}=\rho(\mathbf{x}),\left.\quad P_{-} \psi\right|_{x_{0}=T}=\rho^{\prime}(\mathbf{x})
$$

and

$$
\left.\bar{\psi} P_{-}\right|_{x_{0}=0}=\bar{\rho}(\mathbf{x}),\left.\quad \bar{\psi} P_{+}\right|_{x_{0}=T}=\bar{\rho}^{\prime}(\mathbf{x})
$$

with projectors

$$
P_{ \pm}=\frac{1}{2}\left(1 \pm \gamma_{0}\right)
$$

The spatial fields $\rho, \rho^{\prime}, \bar{\rho}, \bar{\rho}^{\prime}$ are formal Grassmann valued sources which, after possible differentiations will always be set to zero. The partition function (45) now depends on all boundary fields $\mathcal{Z}=\mathcal{Z}\left(C^{\prime}, \bar{\rho}^{\prime}, \rho^{\prime} ; C, \bar{\rho}, \rho\right)$ and is given by the finite volume path integral

$$
\mathcal{Z}=\int D U D \psi D \bar{\psi} \exp \left[-S_{\mathrm{W} ; \mathrm{Wsf}}(U, \psi, \bar{\psi})\right]
$$

which as an example we have written with the plaquette action and plain Wilson fermions and the (here suppressed) boundary fields enter into the action.

As mentioned before we want to eliminate $\mathrm{O}(a)$ artefacts throughout. We know already that this requires the clover term to be added to the bulk quark action. But, as for the gluons, the presence of boundaries allows for new terms in the Symanzik action that have to be canceled by corresponding improvement terms. A discussion of the relevant dimension four densities integrated

\footnotetext{
${ }^{3}$ It has become customary to call this twisted boundary conditions, although it should not be confused with t'Hooft type twisted boundary conditions referring to planes of a torus.
} 

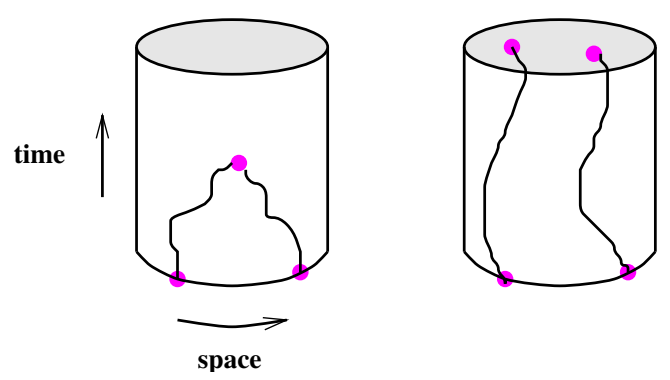

Figure 5: Correlation functions in the Schrödinger functional. On the left we show boundary-to-bulk correlation functions such as $f_{\mathrm{P}}$ and on the right the boundary-to-boundary correlation function $f_{1}$.

over the $x_{0}=0, T$ time slices is found in [16] together with their reduction due to the on-shell conditions. The upshot is a rather simple modification of the quark action by a contribution at $x_{0}=a$ given by

$$
\left(\tilde{c}_{t}-1\right) a^{4} \sum_{\mathbf{x}}\left[\bar{\psi} P_{+} D_{0}^{*} \psi+\bar{\psi} \overleftarrow{D}_{0}^{*} P_{-} \psi\right]
$$

and another such term with the same coefficient at the other boundary. Again $\tilde{c}_{t}$ has to be tuned and possesses a perturbative expansion

$$
\tilde{c}_{t}\left(g_{0}\right)=1+\tilde{c}_{t}^{(1)} g_{0}^{2}+\ldots
$$

Additional terms involving spatial boundary terms do not contribute for constant sources $\rho, \ldots$, to which our applications will be restricted, or can be absorbed into rescaling the sources which undergo multiplicative renormalization anyway. If in the following we refer to the Sheikholeslami Wohlert improved quark action $S_{\mathrm{SWsf}}$ for the SF, we assume the $\tilde{c}_{t}$ terms to be included, too.

\subsection{Boundary quark operators}

We introduce 'functional' differentiation operators for the boundary sources

$$
\begin{aligned}
\zeta(\mathbf{x})=a^{-3} \frac{\partial}{\partial \bar{\rho}(\mathbf{x})}, \quad \bar{\zeta}(\mathbf{x})=-a^{-3} \frac{\partial}{\partial \rho(\mathbf{x})} \\
\zeta^{\prime}(\mathbf{x})=a^{-3} \frac{\partial}{\partial \bar{\rho}^{\prime}(\mathbf{x})}, \quad \bar{\zeta}^{\prime}(\mathbf{x})=-a^{-3} \frac{\partial}{\partial \rho^{\prime}(\mathbf{x})} .
\end{aligned}
$$

If they contribute to an observable $O$ expectation values are meant in the sense

$$
\begin{gathered}
\langle O\rangle= \\
\left\{\frac{1}{\mathcal{Z}} \int D U D \psi D \bar{\psi} O \exp [-S(U, \psi, \bar{\psi})]\right\}_{\bar{\rho}^{\prime}=\rho^{\prime}=\bar{\rho}=\rho=0}
\end{gathered}
$$

where $S$ is any of our lattice actions and it contains the boundary values. Inspection of any of these actions shows that differentiation with respect to $\rho(\mathbf{x})$ for example leads to single insertions of the dynamical quark fields with suitable parallel transporters. They transform contragrediently to $\rho(\mathbf{x})$, i. e. like $\bar{\rho}(\mathbf{x})$. Hence the various $\zeta, \ldots$ can be contracted to form boundary currents like for example

$$
O^{a}=a^{6} \sum_{\mathbf{u}, \mathbf{v}} \bar{\zeta}(\mathbf{u}) \gamma_{5} \frac{1}{2} \tau^{a} \zeta(\mathbf{v})
$$

and the corresponding primed operator at the other boundary. They allow to form the SF standard correlation functions

$$
f_{\mathrm{P}}\left(x_{0}\right)=-\frac{1}{3}\left\langle P^{a}(x) O^{a}\right\rangle
$$

and the boundary to boundary constant

$$
f_{1}=\left\langle O^{\prime a} O^{a}\right\rangle,
$$

which are illustrated in figure 5. We may use these correlation functions to define a convenient normalization condition for $Z_{\mathrm{P}}$ in (36) by postulating

$$
Z_{\mathrm{P}}=\text { const. } \sqrt{f_{1}} / f_{\mathrm{P}}(T / 2)
$$

where multiplicative renormalization factors of the boundary fields cancel. Some choice has to be adopted for the kinematical parameters: aspect ratio $T / L$, the sources, angles $\theta_{k}$ and for $x_{0} / T$. The constant is then chosen such that $Z_{\mathrm{P}}=1$ holds at tree level. As emphasized before we would like to establish the SF as a massless renormalization scheme. We therefore want to tune the bare masses $m_{0}$ to their critical value. At least in perturbation theory this is possible as the SF supplies an infrared regulator by providing a mass gap of order $L^{-1}$.

A similar standard matrix element involving the axial current is defined by

$$
f_{\mathrm{A}}\left(x_{0}\right)=-\frac{1}{3}\left\langle A_{0}^{a}(x) O^{a}\right\rangle
$$

which allows us to define a bare improved PCAC mass

$$
m=\frac{\tilde{\partial}_{0} f_{\mathrm{A}}\left(x_{0}\right)+c_{\mathrm{A}} a \partial_{0}^{*} \partial_{0} f_{\mathrm{P}}\left(x_{0}\right)}{2 f_{\mathrm{P}}\left(x_{0}\right)} .
$$

In the next step this leads to the renormalized running mass

$$
\bar{m}(L)=m \frac{Z_{\mathrm{A}}\left(1-b_{\mathrm{A}} a m_{q}\right)}{Z_{\mathrm{P}}\left(1-b_{\mathrm{P}} a m_{q}\right)} .
$$

If all improvement and renormalization factors assume their correct values $\bar{m}$ is well defined up to $\mathrm{O}\left(a^{2}\right)$ uncertainties. This is visible as small variations under changes of kinematic parameters ( $x_{0}$ for example) on which the lattice but not the continuum results depend. 


\section{Gradient flow}

The finite volume SF coupling defined in the previous section has been the key tool for the ALPHA collaboration's effort to relate low and high energies in QCD for many years. Recently the technique of the gradient flow [35] has given access to a large class of alternative observables that may serve in the same function. It has turned out in the meantime that it is a good strategy to keep SF boundary conditions, typically with zero boundary fields, to define massless finite size schemes based on gradient flow (GF) couplings [36]. They will be found superior in practise to the SF coupling at intermediate energy scales, but not at the perturbative end of the scale evolution. In addition, perturbation theory is more developed and also easier for the traditional SF coupling where the three-loop term of the $\beta$-function as well as some two loop results for improvement coefficients like $c_{t}$ are available (for $S_{\mathrm{W} ; \mathrm{SW} \text { sf }}$ ) [37]. Therefore a good way forward is to use a particular optimized GF coupling for the lower energy part of the scale evolution and, at an intermediate energy, to match it to the SF coupling which is then run up to perturbatively high energies. This promises the best over-all precision and is thus a good reason to also exploit gradient flow couplings and review them here.

\subsection{Gradient flow in the continuum theory}

In the continuum Yang Mills theory we follow [35] and grow a one parameter $(t)$ family of gauge potentials $B_{\mu}(t, x)$ starting in an arbitrary four dimensional potential $A_{\mu}(x)$ which is taken as an initial value

$$
B_{\mu}(t=0, x)=A_{\mu}(x) .
$$

The trajectory is defined by imposing the first order flow equation

$$
\frac{\partial B_{\mu}(t, x)}{\partial t}=-\frac{\delta S_{\mathrm{YM}}[B]}{\delta B_{\mu}(t, x)}
$$

where $S_{\text {YM }}$ is the usual Euclidean Yang Mills action evaluated for potentials at any $t$ value, which will be referred to as flow 'time'. The action is lowered with growing $t$ as we move along the steepest descent direction of the action. Thus along the trajectory the initial field $A_{\mu}$ gets smoothed.

It is most remarkable that correlation functions at finite $t$ are finite without additional renormalizations beyond those of the four dimensional theory. In [38] this has been shown to all orders of perturbation theory by performing an analysis based on Feynman rules of a five dimensional theory where $t$ is an extra coordinate with the dimension of a squared length. The five dimensional action is constructed with additional Lagrange multipliers in such a way that the flow equation appears as a constraint in the functional integral. Similar techniques have been known for some time in the related field of stochastic quantization [39].

A particularly striking new finite observable is the local action density

$$
E(t)=-\frac{1}{2} \operatorname{tr}\left(G_{\mu \nu}(t, x) G_{\mu \nu}(t, x)\right),
$$

where $G_{\mu v}(t, x)$ is the SU(3) curvature tensor of the potential $B_{\mu}(t, x)$, which has a finite expectation value at positive flow time. In [35] the leading order perturbation theory result

$$
\left\langle t^{2} E(t)\right\rangle=\frac{3}{16 \pi^{2}} \bar{g}_{\overline{\mathrm{MS}}}^{2}(\mu)+\mathrm{O}\left(\bar{g}_{\overline{\mathrm{MS}}}^{4}\right), \quad \mu=\frac{1}{\sqrt{8 t}}(67)
$$

has been derived. To leading order the length $\sqrt{8 t}$ is the radius over which the initial values $A_{\mu}(x)$ are smoothed by solving (65) and is thus a natural scale for the $\overline{\mathrm{MS}}$ coupling to expand in. This shows that up to a computable normalization factor the combination $t^{2}\langle E(t)\rangle$ can be regarded as a renormalized coupling constant which - in contrast to $\bar{g}_{\overline{\mathrm{MS}}}$ - is however defined also beyond perturbation theory. In section 2 we have explained the advantages for our purposes in defining coupling constants running with the finite size. This remains true for GF couplings. We want however to stay with couplings running with only a single scale and therefore tie together $t, L$ by setting

$$
\sqrt{8 t}=c L
$$

with a fixed proportionality constant $c$ of order unity. Its value and the details of the finite size boundary conditions together form part of the coupling definition and each choice represents a different scheme. These different variants are in finite relations with each other and a particular choice will be determined by practical considerations. In QCD with $N_{\mathrm{f}}$ quark species the flow (65) is unchanged with only $S_{\mathrm{YM}}$ appearing. Also the leading order result (67) is $N_{\mathrm{f}}$ independent.

We remark that the result (67) indicates that GF couplings are not technically simple in perturbation theory. The normalization factor in defining a renormalized coupling constant via the bare coupling is usually given by a trivial tree level result. For GF couplings a diffusion process corresponding to the free flow equation has to be solved to normalize $E(t)$ and this calculation is already comparable to the evaluation of one-loop diagrams. The next correction would resemble a two loop calculation etc. 


\subsection{Gradient flow on a finite lattice}

In principle the transcription to the lattice is straight forward, but many choices have to be made that differ in their lattice artefacts. First of all, a lattice discretization for the action in the four dimensional path integral has to be chosen as before, with which configurations $U(x, \mu)$ are generated. In addition, the action whose gradient is taken in the flow equation must be discretized, and this need not necessarily be the same discretization. Finally, a lattice substitute for the action density must be picked.

On the lattice the gluon field is Lie group rather than algebra valued. This requires standard changes to (65). Instead of $B_{\mu}(t, x)$ we now introduce a family of group valued link field $V(t, x, \mu)$ by

$$
V(t=0, x, \mu)=U(x, \mu)
$$

and

$$
a^{2} \frac{\partial V(t, x, \mu)}{\partial t} V(t, x, \mu)^{-1}=-g_{0}^{2} \partial_{x, \mu} S[V] .
$$

Here $S$ is any lattice gluon action (e. g. $S_{\mathrm{W}}$ or $S_{\mathrm{LW}}$ ). The Lie algebra valued left derivative gradient of a scalar function on the group is given by (suppressing here the link index $x, \mu$ )

$$
\partial f[U]=\left.\sum_{a} T^{a} \frac{d}{d s} f\left[\exp \left(s T^{a}\right) U\right]\right|_{s=0}
$$

with $T^{a}$ being a basis of group generators. Such a lattice version of the gradient flow based on $U(x, \mu)$ fields has been first written down in [35].

In [40] a careful assessment of lattice artefacts of gradient flow observables has been reported. We do not review any details which proceed via an analysis of the five dimensional Symanzik effective theory. The remarkably simple result is that the major part of all $\mathrm{O}\left(a^{2}\right)$ coming from the flow equation is cancelled if we invoke the tree level improved Lüscher Weisz action for the gradient flow with just one additional term and replace (70) by

$$
\begin{aligned}
& a^{2} \frac{\partial V(t, x, \mu)}{\partial t} V(t, x, \mu)^{-1}= \\
& -g_{0}^{2}\left(1+\frac{a^{2}}{12} D_{\mu}^{*} D_{\mu}\right) \partial_{x, \mu} S_{\mathrm{LW}}[V],
\end{aligned}
$$

where the covariant derivatives defined in (15), (16) have to be taken with $V(t, x, \mu)$ here. The discretized energy density to form $E$ has to be read off from the density in $S_{\mathrm{LW}}$ (27). It has to be remembered however that the usual $\mathrm{O}\left(a^{2}\right)$ effects of the four dimensional theory are unchanged, and it is just the additional source from the flow that one tries to control here. More numerical experiments with the rather recent proposal (72) are required.

To evaluate flow observables in simulations some solver for first order equations in time has to be employed. This will require also a discretization of flow time, but this step-size error can be kept at a negligible level. In appendix C of [35] a third order Runge Kutta integrator on Lie groups has been proposed in a fully explicit form that is easy to implement. It is reported that a step-size $\epsilon=0.01$ in $t / a^{2}$ is accompanied by errors of $10^{-6}$ in link variables. A more sophisticated variant in [36] automatizes the choice of $\epsilon$ by combining the previous Runge Kutta integrator with adaptive step-size control.

To define a finite size scheme based on a GF coupling given by the expectation value of $\langle E(t)\rangle$ boundary conditions have to be specified. The first attempt to do so was made for simple periodic boundary conditions in [41]. It has been known for a long time that Yang Mills theory on a small torus is complicated due to the presence of non-Gaussian fluctuation modes (see refs. in [36]). This leads to a small coupling expansion of torus based finite size couplings that is non-analytic in $g_{0}^{2}$. As discussed in section 4 the SF in contrast has a unique minimum with purely Gaussian fluctuations up to exact gauge modes that have to be fixed in the usual way and then no non-analyticity arises. Moreover, zero field SF Dirichlet boundary conditions provide an infrared regulator and hence the additional bonus is that the quark masses can be set to zero and we continue to have a massless scheme as with the SF coupling before.

To achieve the exact normalization on the lattice $\mathcal{N}^{-1} t^{2}\left\langle E\left(t, x_{0}\right)\right\rangle=g_{0}^{2}+\mathrm{O}\left(g_{0}^{4}\right)$ the factor $\mathcal{N}$ has to be calculated for the finite lattices in use by taking into account all details like choice of discretization, boundary conditions, the ratio $c$ in (68) etc. Note that in the SF there also is the indicated $x_{0}$ dependence and the obvious standard choice is to take $x_{0}=T / 2$ here. Depending on the action in use $\mathcal{N}$ is calculated in closed form or numerically, involving a finite momentum sum, and the required values may be tabulated once and for all. The effort in any case is negligible.

In figure 6 we have a first look at the cutoff dependence of a step scaling function of a GF coupling a la [36]. Some details of this experiment are: $N_{\mathrm{f}}=0, T=$ $L, c=0.3$ with the actions $S_{\mathrm{LW}}$ for the $U$ sampling, $S_{\mathrm{W}}$ in the flow equation (70) and a clover-discretized $E$. We see a nice extrapolation to the continuum limit but also a non-negligible slope and a clear break off from an approximate asymptotic $a^{2}$ behavior beyond $a / L \geq 1 / 8$ (vertical dashed line). More detailed investigations of 


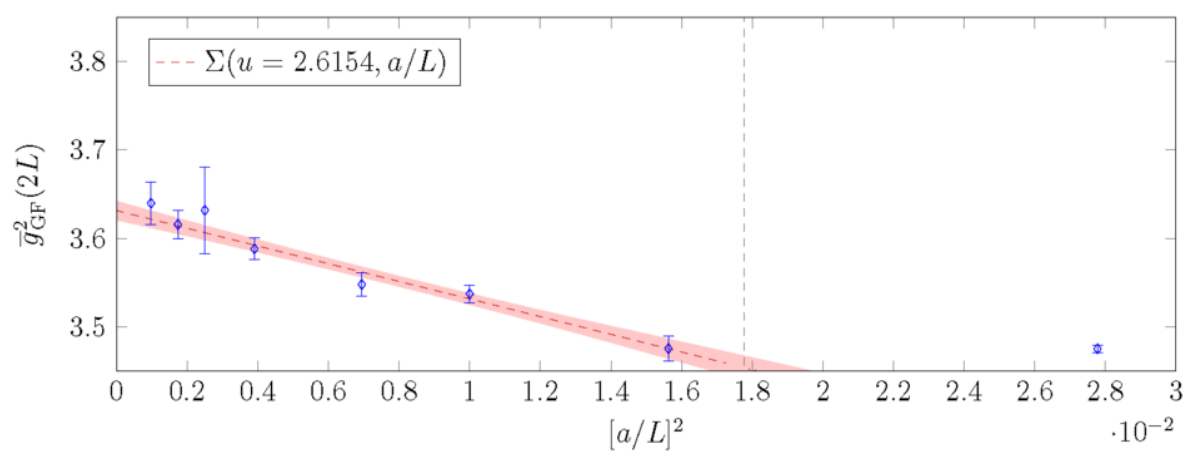

Figure 6: Step scaling function of a gradient flow coupling for $N_{\mathrm{f}}=0$.

the cutoff effects of GF couplings are underway and the improved flow equation (72) is presently simulated.

\section{Some details on simulation algorithms}

The whole physics programme reviewed in this article depends on the availability of appreciable (parallel) computer resources and their optimal exploitation with efficient algorithms (see [42] for a review). While the resources mainly have to be provided by outside supercomputer centers, algorithmic research and development is a major occupation of the physics collaborations. Usually useful ideas can only be advanced if the physics that one tries to extract is understood in detail. This section is therefore meant to reflect some of these efforts without being able to cover the whole field.

\subsection{Hybrid Monte Carlo}

Algorithms of the Hybrid Monte Carlo (HMC) class are at present the only known method that is able to cope with QCD at $N_{\mathrm{f}}>0$, although for $N_{\mathrm{f}}=0$ and for other bosonic lattice field theories much more efficient algorithms exist. The problem is the nonlocal contribution of the fermion determinant in (20).

The HMC $[43,44]$ method is based on molecular dynamics. There for each link $U(x, \mu)$ a conjugate momentum $\pi(x, \mu)$ in the Lie algebra is introduced and a Hamiltonian is considered

$$
\mathcal{H}[\pi, U]=-\sum_{x \mu} \operatorname{tr}\left(\pi^{2}(x, \mu)\right)+S[U]
$$

with some lattice action $S[U]$ that plays the role of a potential here. We now consider an enlarged ensemble with a partition function given by the path integral

$$
\tilde{Z}=\int D \pi D U \mathrm{e}^{-\mathcal{H}[\pi, U]}
$$

where the $\pi$ fields are integrated with the obvious measure over their 8 dimensional real vector space for each link. It is clear that observables depending only on $U$ assume the desired expectation values in this ensemble as the Gaussian $\pi$ integrations simply factorize out.

The HMC update procedure for the enlarged ensemble is now given by the following sequence of steps:

- a complete field of independently Gaussian distributed $\pi(x, \mu)$ is drawn at random,

- with the given configuration $(\pi, U)$ as initial values at $t=0$ Hamilton's equations are solved in molecular dynamics time ${ }^{4} t$

$$
\begin{aligned}
& \dot{\pi}(x, \mu)=-\partial_{x, \mu} S[U] \\
& \dot{U}(x, \mu)=\pi(x, \mu) U(x, \mu)
\end{aligned}
$$

up to some trajectory length $t=\tau$. In practice this is done with some inexact discretized integrator with a finite step size that has to be exactly time reversible,

- only due to step size errors of the integrator the Hamiltonian between $t=0$ and $t=\tau$ will change $\mathcal{H} \rightarrow \mathcal{H}+\Delta \mathcal{H}$. The end configuration is taken as a successor of the initial one with the Metropolis acceptance probability $\min (1, \exp (-\Delta \mathcal{H}))$. In the case of rejection the old configuration remains unchanged.

An important point here is that no zero step size limit is needed to prove detailed balance for the HMC. In practice however the step size $\tau / N_{\text {step }}$ has to be small enough to make the rejection rate small, around $10 \%$ for example in practice.

\footnotetext{
${ }^{4}$ This $t$ has nothing to do with the flow time before.
} 
With two dynamical quark species for $S$ an effective action like

$$
S_{\mathrm{eff}}[U]=S_{\mathrm{W}}[U]-\ln \operatorname{det}\left(M^{2}\right)
$$

should be taken that includes the fermion determinant. We have introduced here the usual lattice parameterization for the Dirac matrix (for one flavor)

$$
M=2 \kappa a\left(D+m_{0}\right)
$$

with the hopping parameter

$$
\kappa=\frac{1}{8+2 a m_{0}},
$$

where $D$ will be $D_{\mathrm{Sw}}$ in most cases here. Its nonnegative squared determinant corresponds to two degenerate quark species as in the $N_{\mathrm{f}}=2$ theory. Other cases complicate simulations further and will only be mentioned later. The advantage of HMC in this context is that in $\partial_{x, \mu} S_{\text {eff }}$ only the response of $S_{\text {eff }}$ to infinitesimal rather than finite moves in $U$, which enters into matrix elements of $M$, is required. The derivative of the $\ln$ det contribution in $S_{\text {eff }}$ leads to the necessity to control arbitrary matrix elements of the non-sparse matrix $M^{-1}$. In four dimensional QCD on large lattices also this is impractical. Therefore the additional trick of introducing pseudofermions is needed that we discuss next. With them we shall only have to solve systems of linear equations with the Dirac matrix as coefficients for which highly efficient tools exist.

\subsection{Pseudofermions}

A pseudofermion is a complex field $\phi(x)$ which carries the same Dirac and color indices as one quark species. The squared determinant can now be represented by a Gaussian path integral

$$
|\operatorname{det}(M)|^{2}=\int D[\phi] \exp \left(-S_{\mathrm{pf}}[\phi]\right)
$$

with

$$
S_{\mathrm{pf}}[U, \phi]=\sum_{x}\left|M^{-1} \phi\right|^{2} .
$$

A number of comments are in order:

- $D[\phi]$ means independent integration over the real and imaginary part of all components and some (later irrelevant) normalization,

- due to the inversion of $M$ the action of $\phi(x)$ is highly nonlocal,
- for a pair of degenerate improved SW quarks there is no sign problem although the Dirac operator $D_{\text {Sw }}$ has complex eigenvalues. This is so since the determinant is real because of the relation

$$
M^{\dagger}=\gamma_{5} M \gamma_{5} .
$$

With the pseudofermion representation of the determinant the HMC Hamiltonian becomes

$$
\mathcal{H}[\pi, U, \phi]=\frac{1}{2}(\pi, \pi)+S_{\mathrm{W}}[U]+S_{\mathrm{pf}}[U, \phi]
$$

with a scalar product notation for the $\pi$ part and Wilson gluons as an example. Note that we have not introduced momenta conjugate to $\phi$. As they are Gaussian a correctly distributed $\phi$ configuration can be trivially drawn (for given $U$ ) by applying once the Dirac operator in

$$
\phi=M \eta \text {. }
$$

Here $\eta$ has the same indices (color, Dirac) as $\phi$ and each component is an independent Gaussian random number with zero mean and unit variance. Now each HMC trajectory starts with a global choice of $\pi$ and $\phi$ and then an evolution in molecular dynamics time $t=0, \ldots, \tau$ and the accept/reject step. The force during the evolution stems from the gluon action and from $S_{\mathrm{pf}}$. The derivative (71) of the gluon action for a given link is given by a sum of small Wilson loops, 'staples' for $S_{\mathrm{W}}$, that require small computation time. The derivative of $S_{\mathrm{pf}}$ derives from the variation of the discretized Dirac operator $M \rightarrow M+\delta M$ as the $U(x, \mu)$ entering its matrix elements change. It is given by

$$
\delta S_{\mathrm{pf}}=-\sum_{x} \chi^{\dagger} M^{-1} \delta M \chi+c . c .
$$

with

$$
M \chi=\phi .
$$

For each computation of the pseudofermion force we thus have to solve two linear systems involving the Dirac matrix. It is this step - solving the lattice Dirac equation with given right hand sides - which consumes by far the most time in QCD simulations with the HMC algorithm.

Obviously it is worthwhile to optimize the linear equation solvers used with HMC as far as possible and a corresponding effort has been devoted to this issue by the lattice community. As the Dirac matrix in any of the discretizations discussed here is sparse, iterative Krylov space solvers are the method of choice. At first the simple and robust conjugate gradient method [45] has been widely used. A large number of improvements 
have been developed over the years with incremental small speed-up factors. A kind of breakthrough with regard to the slowing down for small quark masses has been achieved more recently with low mode deflation [46, 47] and with multigrid methods [48].

Also the integrators used for Hamilton's equations inside of HMC allow for improvement. One example is the multiple time step size technique [49]. It is based on the observation that the pseudofermion force, that is expensive to compute, is usually much smaller than the gluonic force. The former would therefore admit larger time steps leading to fewer evaluations. In [49] a modified leapfrog integrator is proposed that allows for different step sizes for the two components while still maintaining the required reversibility. Other possibilities are to minimize the higher order step size errors and a popular integrator in this respect has been proposed in [50].

\subsection{Hasenbusch preconditioning}

Hasenbusch [51] has proposed a simple factorization of the two flavor determinant

$$
|\operatorname{det}(M)|^{2}=|\operatorname{det}(\tilde{M})|^{2} \times\left|\operatorname{det}\left(M \tilde{M}^{-1}\right)\right|^{2}
$$

which has been further investigated in [52]. Above, $\tilde{M}$ contains a larger mass $(\tilde{\kappa}<\kappa)$. One pseudofermion is introduced now for each factor with an action

$$
S_{\mathrm{pf}}=\left(\tilde{M}^{-1} \phi_{1}, \tilde{M}^{-1} \phi_{1}\right)+\left(\tilde{M} M^{-1} \phi_{2}, \tilde{M} M^{-1} \phi_{2}\right) .
$$

With some tuning of $\tilde{\kappa}$ the condition numbers of both factors can be lower than the one of the original $M$. As a consequence the forces are of smaller magnitude with smaller fluctuations and the step size can be increased by a factor two or so. It turned out that it was important to tune $\tilde{\kappa}$ properly [53] and also more than two factors can be introduced.

\subsection{Simulation of nondegenerate quarks, $N_{\mathrm{f}}>2$}

All simulation techniques and improvements discussed so far have exploited that the a fermion determinant was squared for two degenerate fermions. Its weight could not go negative and the factorization was useful for introducing a pseudofermion. To model QCD more precisely the inclusion of the strange (and ultimately also charmed) quarks becomes necessary.

The strange quark implies an additional weight $\operatorname{det}\left(M_{s}\right)$ to be included in the Boltzmann factor where the Dirac matrix $M_{s}$ includes the strange mass. Following [42] and [54] we start from the trivial factorization

$$
\operatorname{det}\left(M_{s}\right)=W_{s} \operatorname{det}\left(R^{-1}\right), \quad W_{s}=\operatorname{det}\left(M_{s} R\right) .
$$

The goal is to construct $R$ such that
- $R \approx\left(M_{s}^{\dagger} M_{s}\right)^{-1 / 2}$ and $W_{s} \approx 1$,

- $R$ can be represented by one or several pseudofermions.

A well known solution is the rational approximation of degree $m$ [55]

$$
R=C \prod_{k=0}^{m-1} \frac{M_{s}^{\dagger} M_{s}+\omega_{k}^{2}}{M_{s}^{\dagger} M_{s}+v_{k}^{2}} .
$$

If one decides on a spectral interval $\left(\epsilon^{2}, r^{2}\right)$ of $M_{s}^{\dagger} M_{s}$ on which $R$ approximates the inverse square root then there is the well defined Zolotarev algorithm to construct those $C,\left\{\omega_{k}\right\},\left\{v_{k}\right\}$ that optimize the approximation quality in a certain norm. In addition the shifts can be taken real, positive and ordered

$$
0<v_{0}<\omega_{0}<v_{1}<\cdots<\omega_{m-1} .
$$

It is now not difficult to see that, using the rational factorization, $\operatorname{det}\left(R^{-1}\right)$ can be represented by one or several pseudofermions. By attaching the subsets of the factors with similar shifts to several pseudofermions, even something similar to mass preconditioning can be achieved.

In spite of a good rational approximation (at moderate degree $m$ ) we still have to take care of the correction factor $W_{s}$. Due to the lack of chiral symmetry the Wilson type lattice Dirac operator has no rigorous spectral cutoff and may in principle even develop zero eigenvalues for some gauge fields occurring in the path integral (Monte Carlo). In such cases $W_{s}$ may not be close to one and therefore important. Such fluctuations are known to become extremely rare once the mass is not too small and additional light quarks are present. They are further suppressed for large volume. For the strange quark $W_{s}$ should be monitored to have only moderate fluctuations around unity. In precisely this case it can be estimated stochastically by

$$
W_{s}=\left\langle\mathrm{e}^{-\left(\eta,\left(R^{-1} M_{s}^{-1}-1\right) \eta\right.}\right\rangle
$$

with Gaussian random fields $\eta$. Its estimator, possibly averaging over several independent $\eta$ fields, may then be included in observables as usual for reweighting.

Clearly, stochastic strange quark reweighting can not account for fluctuations to negative $\operatorname{det}\left(M_{s}\right)$. As indicated this is however not expected to happen for large enough mass and volume. In massless $N_{\mathrm{f}}=3$ simulations for renormalization purposes the finite volume SF boundary conditions have to be monitored via $W_{s}$ to sufficiently stabilize the spectrum. In the applications reviewed here this is indeed the case. 


\subsection{Topological freezing}

The efficiency of any Monte Carlo algorithm depends on the degree in how far it is able to produce at reasonable cost statistically independent lattice field configurations distributed according to the action of the field theory under study. The statistical error of any observable is given by

$$
\sigma^{2}(O)=v(O) \frac{2 \tau_{\mathrm{int}, O}}{N}
$$

In this relation $v(O)$ is the variance of $O$ which is another expectation value. The number of generated estimates $N$ is divided by the integrated autocorrelation time $\tau_{\text {int }, O}$ which summarizes the decorrelation power of the algorithms for a given observable. There also is a maximal autocorrelation time $\tau_{\exp }$ that is related to the spectral gap in the transition probability (Markov) operator of an algorithm. More details on autocorrelations and error analysis can be found in [56] for example.

Each smooth configuration in a continuum Yang Mills theory on a four dimensional torus carries an integer valued topological quantum number $Q$. In the path integral all configurations are summed over and all $Q$ contribute even if large $|Q|$ may have a small relative weight in some situations depending on the physical size of the torus and the observable. The famous selfdual instanton configurations, for example, are classical fields with $Q= \pm 1$. Quantization and in particular the lattice regularization eliminates in the first place all naive arguments based on continuity and topology. It has been argued however [57] that topological winding numbers do effectively imply a decomposition of the space of $U(x, \mu)$ configurations into sectors that are separated by barriers with large Euclidean action. The numerical problem now is that known Monte Carlo algorithms for QCD, including HMC, change configurations in small steps and the probability to change sectors by sequences of such moves gets very small when the continuum limit is approached. As one lowers $a$ this is first seen in steeply growing autocorrelation times. Once these are very large, they are hard to even discover in the statistical error analysis and there is a risk of unnoticed systematic errors. Clearly observables formed in terms of (a lattice approximation) of $Q$ are optimal diagnostic tools to control this problem of topological freezing.

The seriousness of the freezing problem was noticed during the physics programme reviewed here and led to quite some delay. A precise analysis of the problem was given in [58]. Here a special strategy to sharpen the error analysis was proposed that allows for a reliable error estimation in the range of lattice spacings down to $a \approx 0.05 \mathrm{fm}$ where with our standard algorithms and actions freezing starts to become a problem. The idea of the method is to extract the longest autocorrelation time from measuring $Q^{2}$ and $E(t)$ at finite flow time and feed this information into the error analysis of other observables that couple to topology only weekly. Slightly different strategies can be used for the running GF coupling with SF boundary conditions at intermediate volume [59] which however also suffers from freezing.

Finally in [60] a fundamental solution to the problem was given. Open boundary conditions in Euclidean time - while keeping periodicity in the three spatial directions - abolishes the quantization of topological charge and thus the barriers in field space. Charges can so to speak flow in and out of the boundary hyperplanes. In view of the seriousness of the freezing problem, the price of a somewhat more complicated data analysis in the large volume simulations due to the lost time translation invariance seems reasonable to accept. In addition Symanzik improvement has to be modified due to the presence of boundaries in a way that is somewhat familiar from the SF.

For a recent review covering lattice QCD algorithms in more detail than possible here we refer to [61].

\section{Running of coupling and masses}

In this section we review numerical results that have been achieved for the running of the non-perturbatively defined coupling and mass with the finite system size as renormalized scale in the Schrödinger functional.

\subsection{Step scaling function of the $S F$ coupling at $N_{\mathrm{f}}=2$.}

The SF coupling has been defined in (47) and its evolution is analyzed by computing the lattice step scaling function (SSF) (11) and by extrapolating it to $\sigma$. In the numerical investigations it has turned out that it is highly profitable to accelerate the continuum limit of $\Sigma$. We consider the perturbative expansion of the deviation

$$
\frac{\Sigma(u, a / L)-\sigma(u)}{\sigma(u)}=\delta_{1}(a / L) u+\delta_{2}(a / L) u^{2}+\ldots .
$$

For the simulations on which we report here the SF was implemented with the Wilson plaquette gluon action $S_{\text {Wsf }}$ with SW ('clover') improved quarks $S_{\text {SWsf }}$. For this total action the two loop perturbation theory has been studied on the lattice [37] and hence $\delta_{1}, \delta_{2}$ are known for the relevant values of $L / a$. We use them to define a perturbatively two loop improved SSF

$$
\Sigma^{(2)}(u, a / L)=\frac{\Sigma(u, a / L)}{1+\delta_{1}(a / L) u+\delta_{2}(a / L) u^{2}} .
$$




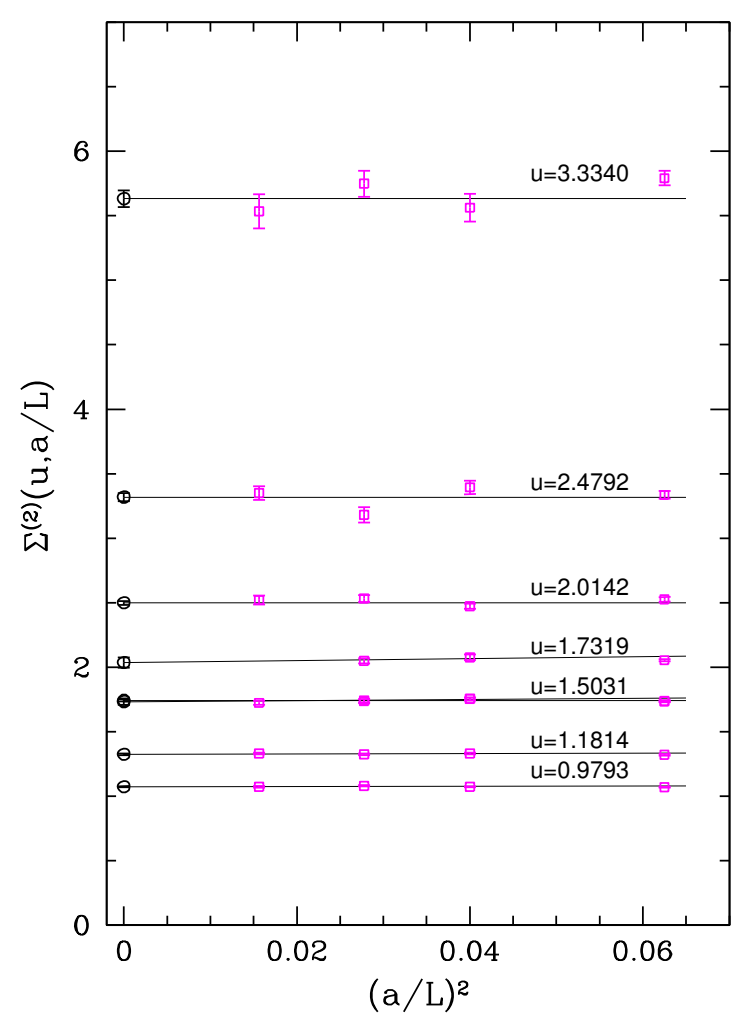

Figure 7: Continuum extrapolations of the Schrödinger functional coupling with $N_{\mathrm{f}}=2$ dynamical quark flavors. Figure from [62].

The very flat and well controlled continuum extrapolation of this quantity is shown in figure 7. Several other technical issues had to be mastered to produce these data. The two bare parameters $g_{0}$ and the bare quark mass (79) $\kappa$ for each $L / a$ have to be tuned to the $u$ values of the series shown together with a vanishing quark mass. This can be achieved only to some limited precision and small corrections have to be applied based on perturbative as well as numerical information. For the quark mass a particular definition $m$ based on a PCAC relation (62) is adopted [62] and it is estimated that a tuning up to $|m L|<0.05$ suffices for the attempted precision.

In figure 8 we find an additional demonstration that the values of $L / a=6 \ldots 12$ have very small discretization errors, at least after our 2-loop improvement of the observable and with the Wilson plaquette gauge action. One can therefore carry out a precise continuum limit with these rather small lattices. On the other hand the figure shows that one cannot take this for granted for any action. Care to take the continuum limit is the most important requirement for a trustable determination of the step scaling functions and ultimately also the $\Lambda$ pa-

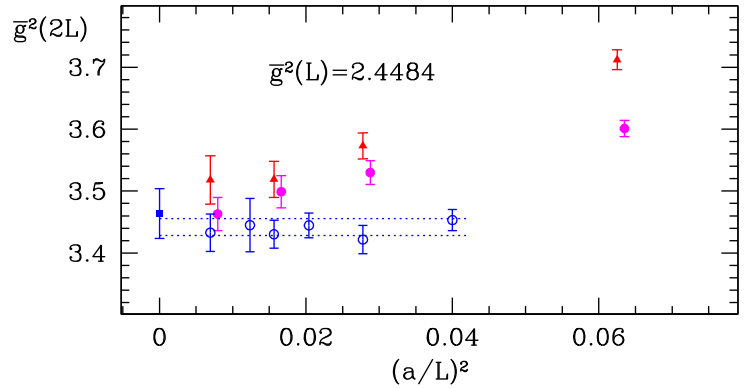

Figure 8: A test of the continuum extrapolations with different actions for $N_{\mathrm{f}}=0$. The data from top (triangles) to bottom (open circles) are for the Iwasaki, the tree level Lüscher Weisz and the Wilson gauge action. Both the boundary improvement of the action and the improvement of the observables have been included. At present this is possible at the 2-loop level for the Wilson gauge action only, and at the 1-loop level in the two other cases. Figure from [29] based on data from $[63,64]$.

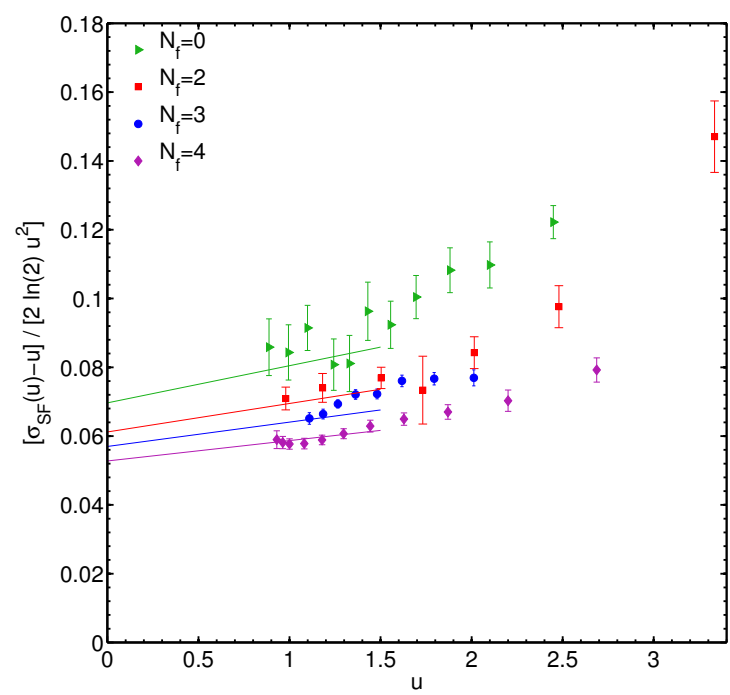

Figure 9: The $N_{\mathrm{f}}$-dependence of the step scaling function of the Schrödinger functional coupling [65]. Non-perturbative results are shown together with the two-loop curves.

rameter.

The continuum extrapolated step scaling function can finally be iterated to construct the non-perturbative running coupling for a number of scale arguments in figure 10. We will see that changing the number of quark flavors from $N_{\mathrm{f}}=0$ to $N_{\mathrm{f}}=2$ does not induce any qualitative changes. The connection from low to high energies is rather smooth and perturbation theory can be trusted in the Schrödinger functional schemes rather precisely at energies $\mu \gtrsim 50 \mathrm{GeV}$ or larger. For the purpose of the comparison we show side by side $N_{\mathrm{f}}=0$ results from [67] where the methods were developed and 

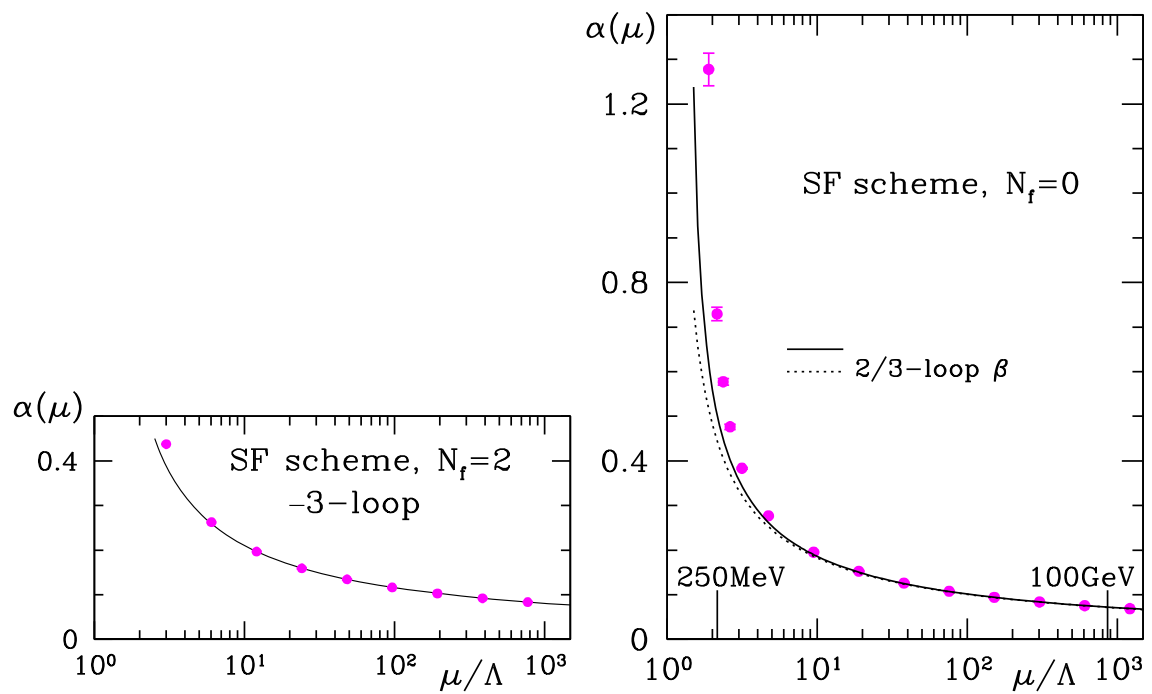

Figure 10: Running coupling for $N_{\mathrm{f}}=2$ compared to $N_{\mathrm{f}}=0$. Figure from [29] based on results of [66, 67, 68, 64, 62].

$N_{\mathrm{f}}=2$ results.

With the continuum step scaling function $\sigma(u)$ under our control for a range $u \in\left(0, u_{\max }\right)$ we can connect the associated scales $\Lambda_{\mathrm{SF}}$ and $L_{\max }$ implicitly defined by the condition

$$
\bar{g}^{2}\left(L_{\max }\right)=u_{\max }=4.484 .
$$

The result, including a complete error analysis, is [69]

$$
L_{\max } \Lambda_{\mathrm{SF}}^{(2)}=0.264(15) .
$$

In terms of the semi-phenomenological scale $r_{0} \approx$ $0.49 \mathrm{fm}$ the estimate $L_{\max } \approx 0.39 \mathrm{fm}$ can be given. This scale is defined [70] in terms of the force $F$ between static quarks by solving

$$
r_{0}^{2} F\left(r_{0}\right)=1.65 .
$$

It is loosely connected to quarkonia models and has in addition been related to other observables in previous lattice simulations. In the next section more direct connections of $L_{\max }$ to phenomenology will be cited.

\subsection{Verification of asymptotic freedom}

Asymptotic freedom is normally taken for granted for QCD with any $0 \leq N_{\mathrm{f}} \leq 6$ and it is certainly a self-consistent property in perturbation theory. Once we have some control beyond perturbation theory we should however remember this situation and analyze our data under this aspect.

From the continuum limit SSF we may form the combination

$$
b_{0}^{\mathrm{eff}}(u)=\frac{\sigma(u)-u}{2 \log 2 u^{2}} .
$$

It is expected to extrapolate to the perturbative coefficient $b_{0}$ at small $u$, with an asymptotic approach that is linear in $u$. In figure 9, where we include additional data for $N_{\mathrm{f}}=0,2,3,4$, we do see the expected behavior. This provides a clear non-perturbative confirmation of asymptotic freedom including the universal perturbative $N_{\mathrm{f}}$ dependence at small coupling.

In perturbation theory asymptotic freedom is lost beyond $N_{\mathrm{f}}=16$ because $b_{0}$ changes its sign. We just remark in passing that we see a considerable effort to determine SSFs for $N_{\mathrm{f}}=8 \ldots 12$ where one expects an almost vanishing $\beta$-function and approximate conformal invariance that may be of phenomenological interest for models of the technicolor variety. Many of these investigations use the methods described in this article, but in a much more difficult situation.

\subsection{Running quark mass}

We now mention also some result for the running of the quark mass which is actually intertwined with the evolution of the coupling constant. The scale dependence of the running mass $\bar{m}(L)$ in (63) derives form the $L$ dependence of $Z_{\mathrm{P}}$ in (60). The renormalization group equation for the mass reads

$$
\mu \frac{d \bar{m}}{d \mu}=\tau(\bar{g}) \bar{m}, \quad \mu=L^{-1}
$$

with $\tau(g)=-8 g^{2} /(4 \pi)^{2}+\mathrm{O}\left(g^{4}\right)$ in perturbation theory. Note that this scale evolution is coupled with the one of $\bar{g}(\mu)$ in (1). To have non-perturbative control also here, 

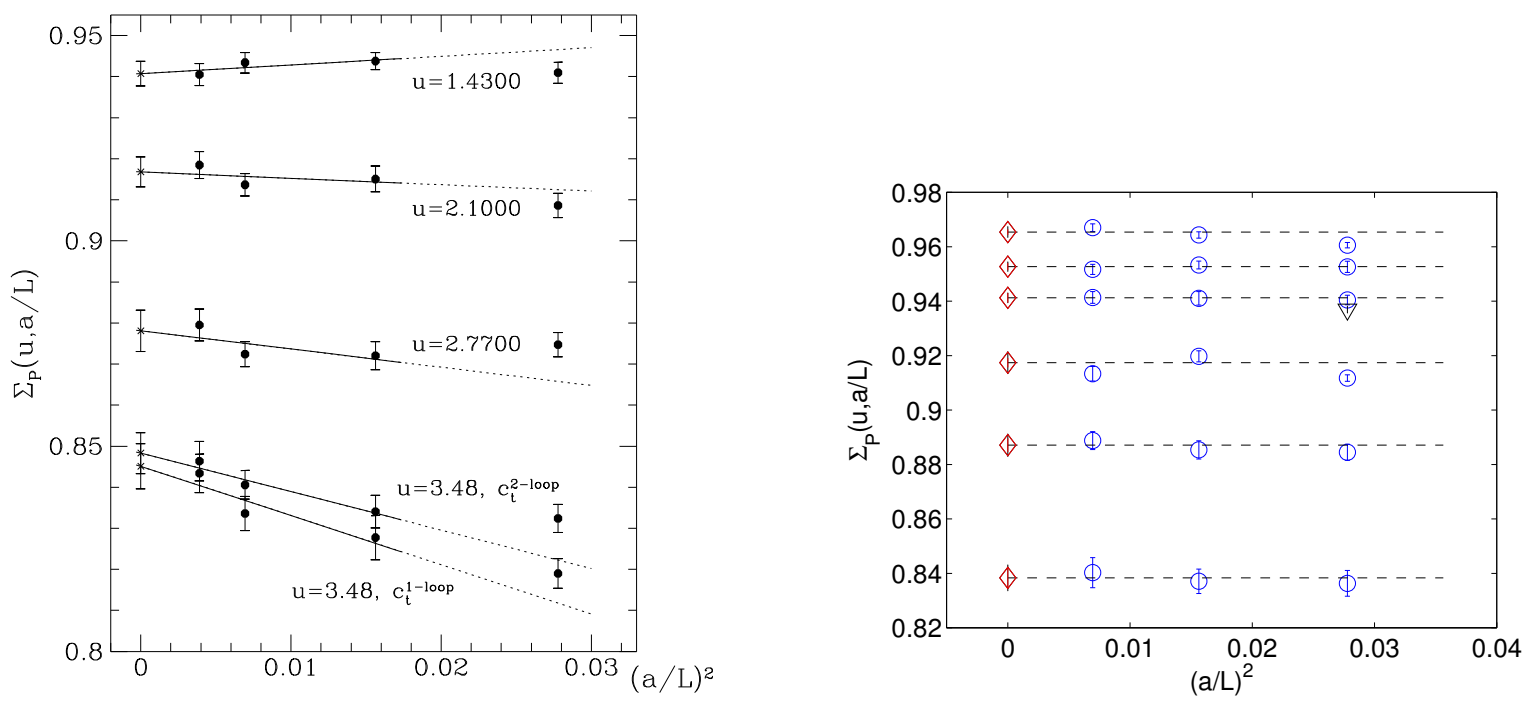

Figure 11: Continuum extrapolations of the step scaling function $\Sigma_{\mathrm{p}}$ in the quenched approximation (left) [67] and with $N_{\mathrm{f}}=2$ dynamical quark flavors [71]. In the right graph the coupling $u$ ranges from $u=0.979$ to $u=3.33$.

we define another step scaling function

$$
\Sigma_{\mathrm{P}}(u, a / L)=\left.\frac{Z_{\mathrm{P}}\left(g_{0}, 2 L / a\right)}{Z_{\mathrm{P}}\left(g_{0}, L / a\right)}\right|_{u=\bar{g}^{2}(L)} .
$$

Also the renormalization group equation for the mass can be converted to an equivalent integral equation

$$
\begin{aligned}
M= & \bar{m}\left(2 b_{0} \bar{g}^{2}\right)^{-d_{0} / 2 b_{0}} \times \\
& \exp \left\{-\int_{0}^{\bar{g}} \mathrm{~d} g\left[\frac{\tau(g)}{\beta(g)}-\frac{d_{0}}{b_{0} g}\right]\right\},
\end{aligned}
$$

where $M$ is the scale independent RGI mass, that is the analogue to the $\Lambda$ parameter in the case of the coupling. The perturbative approximations of $\tau$ and $\beta$ may be used at very high energy (small $L$ in the $\mathrm{SF}$ ) to compute $M, \Lambda$ from $\bar{m}(L)$ and $\bar{g}(L)$. After extrapolating to the continuum limit

$$
\sigma_{\mathrm{P}}(u)=\lim _{a / L \rightarrow 0} \Sigma_{\mathrm{P}}(u, a / L),
$$

see figure 11 , the continuum running mass was constructed. It is shown in figure 12.

\subsection{Optimized strategy}

In figure 9 we have already shown some results for the running of the SF coupling with $N_{\mathrm{f}}=3$. The goal in the present stage of the project is to improve the precision at the same time as having this more realistic number of flavors. As already mentioned at the beginning of section 5 the combination of SF and GF coupling is promising for the precision issue. In figure 13 we show

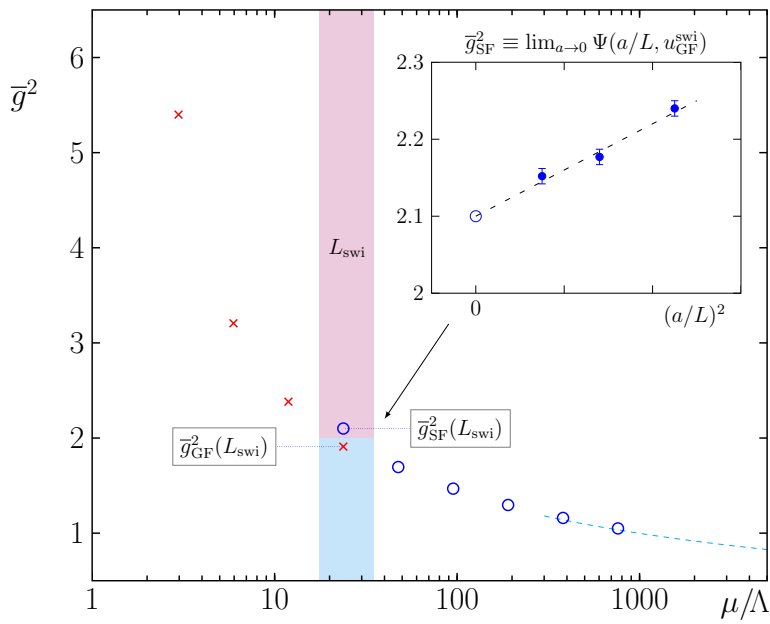

Figure 13: Sketch (no real data) of the overall strategy being employed for $N_{\mathrm{f}}=3[65]$.

a sketch of the overall strategy to use their complementarity and combine the two couplings. We note however, that here we do not yet see real data!

The presently quite different size of the discretization errors in the two observables are seen by comparing figure 8 and figure 6 . We hope that the improved flow equation (72) and the other optimizations reviewed in that subsection will still considerably accelerate the continuum limit of the GF coupling.

Increasing the precision also requires a more precise tuning to the massless theory than before. This is at the 

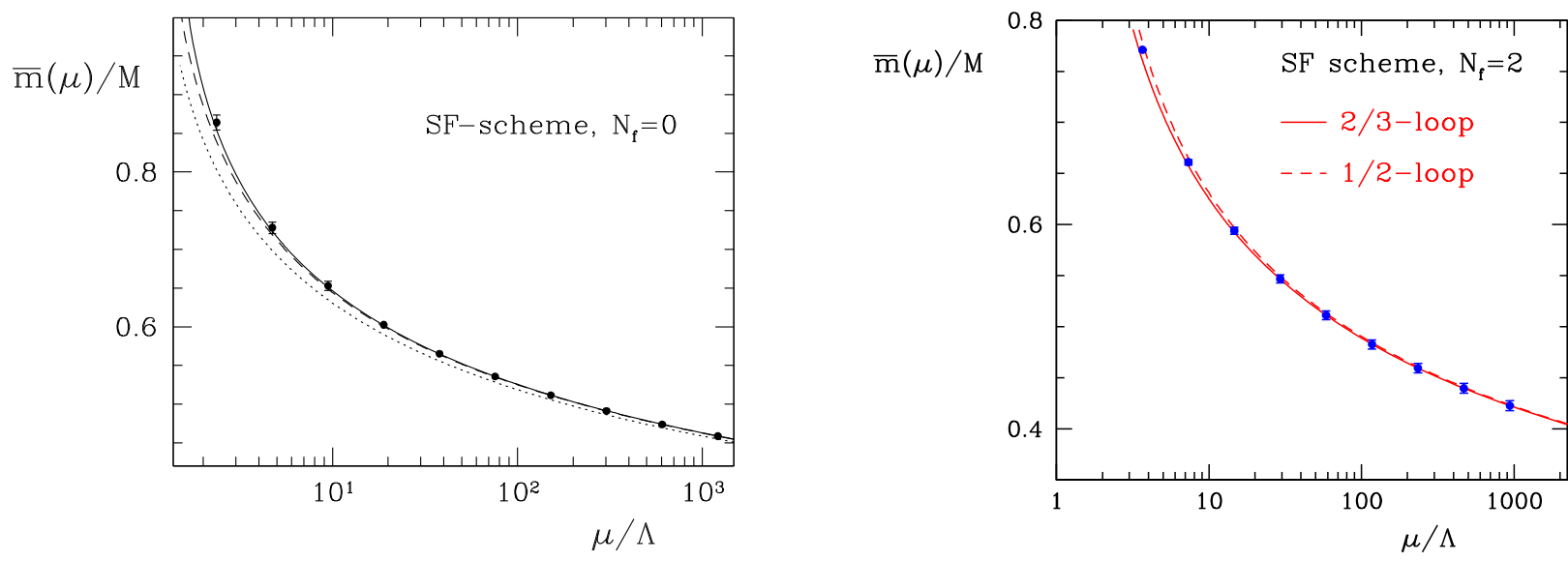

Figure 12: Scale dependence of the quark mass $\bar{m}$ in the quenched approximation (left)[67] and with $N_{\mathrm{f}}=2$ dynamical quark flavors[71]. For $N_{\mathrm{f}}=0$ the dotted, dashed and solid curves are obtained from eqs. (2) and (102) using the 1/2-, 2/2- and 2/3-loop expressions for the $\tau$ - and $\beta$-functions respectively.

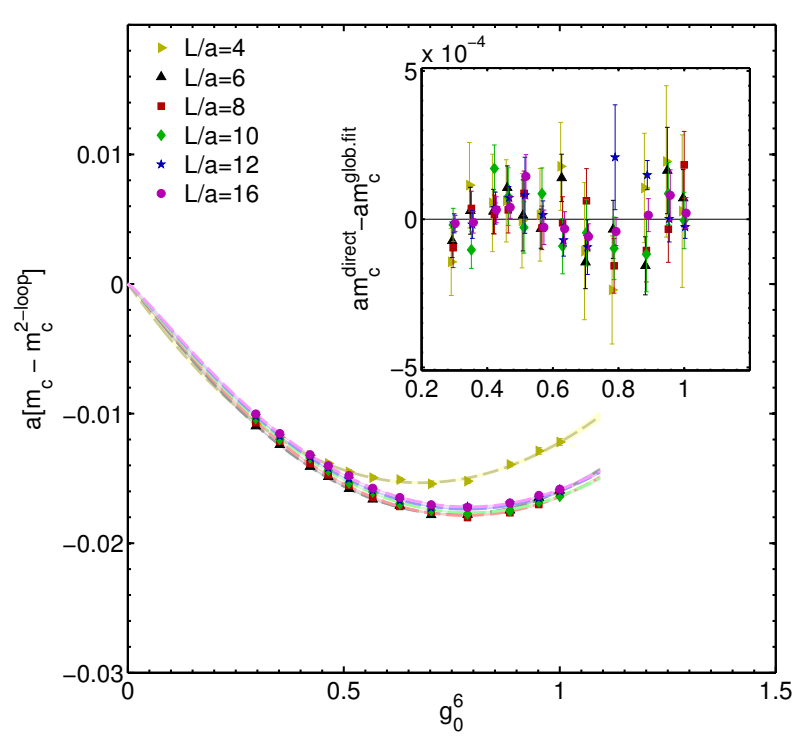

Figure 14: Determination of the critical lines for various values of $L / a$. Note that a big part, the 2-loop expression, is subtracted from the data. Figure from [65].

same time desirable for other projects such as HQET, where the precise knowledge of the relation between $\bar{g}$ and $L$ is used to perform simulations in a volume of fixed physical size. For SW improved Wilson fermions that we use this means that the critical mass in lattice units $\operatorname{am}_{c}\left(g_{0}\right)$ has to be determined to a many digit precision in the relevant range of couplings. For the Wilson gauge action such a result is given in figure 14. For future simulations these data are represented by a smooth interpolating fit formula shown as lines in figure 14.

\section{Large volume simulations}

In this section we review the completed results for $N_{\mathrm{f}}=2$ and some data of the ongoing $N_{\mathrm{f}}=3$ simulations. Quenched computations from the nineties were an extremely useful preparation but will be skipped here.

\subsection{Algorithmic issues}

Here simulations are described that have been run for $N_{\mathrm{f}}=2$ improved Wilson quarks within the Coordinated Lattice Simulation (CLS) consortium.

The algorithmic framework is mostly as discussed in section 6 where it has become clear that the frequent inversion of the lattice Dirac matrix is the dominant numerical task. It is in particular required to evaluate the fermionic driving force for the molecular dynamics evolution in HMC.

Before we have switched to the more efficient Hasenbusch preconditioning (see subsection 6.3), the domain decomposed DD-HMC algorithm [72] had been used initially. This at first very promising variant was ultimately dismissed because of inferior autocorrelations and critical slowing down, but we here still look at some data produced with DD-HMC for comparison and for historical reasons. In figure 15 we see several histories in molecular dynamics time, where the left plots refer to DD-HMC simulations and the right ones (MPHMC) to Hasenbusch mass preconditioning. Components of the fermion forces from two separate pseudofermions are shown and the Hamiltonian violation $\Delta \mathcal{H}$ that enters into the accept step. The spike-like fluctuations that one sees point to algorithmic problems due to 
DD-HMC

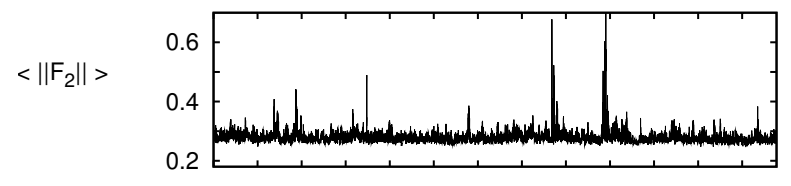

$\Delta \mathrm{H}$

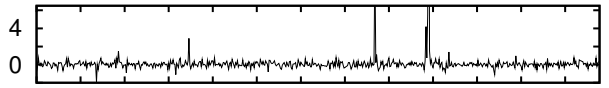

$\operatorname{nax}\left\|F_{2}\right\|$
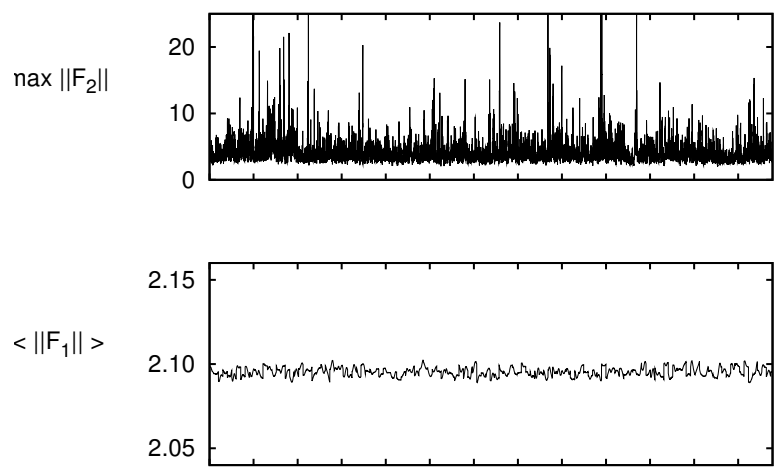

$\operatorname{nax}\left\|F_{1}\right\|$

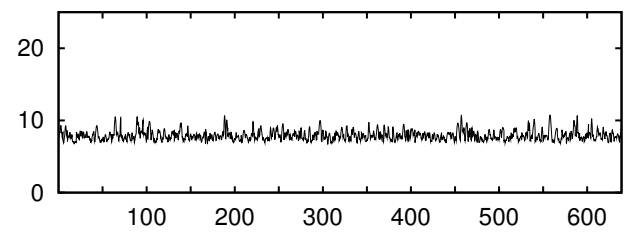

MP HMC
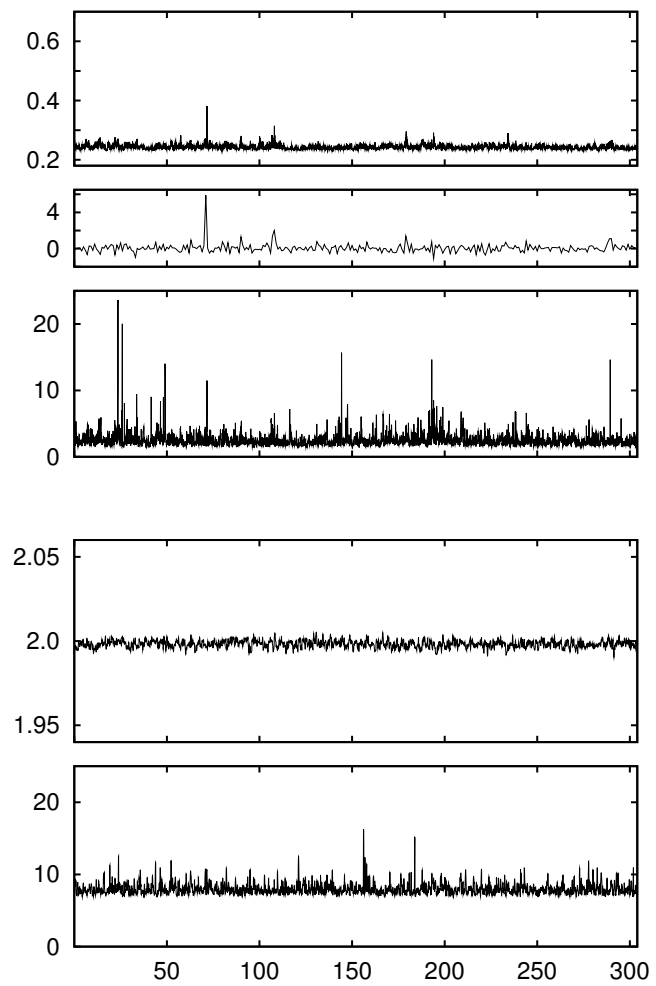

Figure 15: Histories of the energy violation $\Delta H$, as well as maximum and average forces $F_{2}$ and $F_{1}$, for each force update, plotted as a function of the trajectory number. Values corresponding to the DD-HMC algorithm are shown to the left and the integration step-sizes for the two forces relate as $\Delta t_{2}: \Delta t_{1}=1: 6$. The values for MP-HMC are shown in the two right panels and the corresponding ratio of the integration steps is $\Delta t_{1}: \Delta t_{2}=1: 10$. The lattice size is $48 \times 24^{3}$ and $\kappa_{\text {sea }}=0.13625$.

nearly singular Dirac matrices. With them present, one is restricted to small step sizes and finds large iteration numbers for the Dirac solvers which both damage the efficiency. The plots demonstrate the smoother running of the MP-HMC after tuning its parameters in a reasonably close to optimal way [73]. After detailed studies MP-HMC was adopted as the method of choice.

Topological freezing (see subsection 6.5) was the other problem that significantly held back the completion of large volume $N_{\mathrm{f}}=2$ simulations. In the history of the topological charge in figure 16 one sees that the simulations are slowing down dramatically as the lattice spacing is reduced. The observed structures extend over a non-negligible fraction of the length of a typical run. On the other hand, studying the effect of the slow modes of the Monte Carlo algorithm on typical hadronic correlation functions, it was found that these receive only suppressed contributions to their autocorrelation functions. Therefore, for $a \approx 0.05 \mathrm{fm}$ the standard error computation could still be adapted to the situation $[58,74]$. Conservative error estimates are still possible with MC histories which are of the order of 20-100 times the slowest relaxation time of the system, $\tau_{\text {exp }}$.

\subsection{General considerations for scale setting}

In the previous section the energy scales from the perturbative end down to the implicitly defined hadronic scale $L_{\max }$ has been covered for coupling and quark mass running. It remains to connect $L_{\max }$ to a measurable quantity to bring in $\mathrm{MeV}$ units which we call 'scale setting'. After the general discussion in section 2 in principle, if lattice QCD had all dimensionless scale ratios right, any observable would be suitable and the mass of the stable proton would appear particularly natural. In reality, however, many more mundane practical considerations are essential [78] to not give away too much precision in this step.

An obvious demand is that the quantity used for scale setting should be computable on the lattice with good statistical precision (for a given computational effort) and that it should have small systematic errors like cut- 

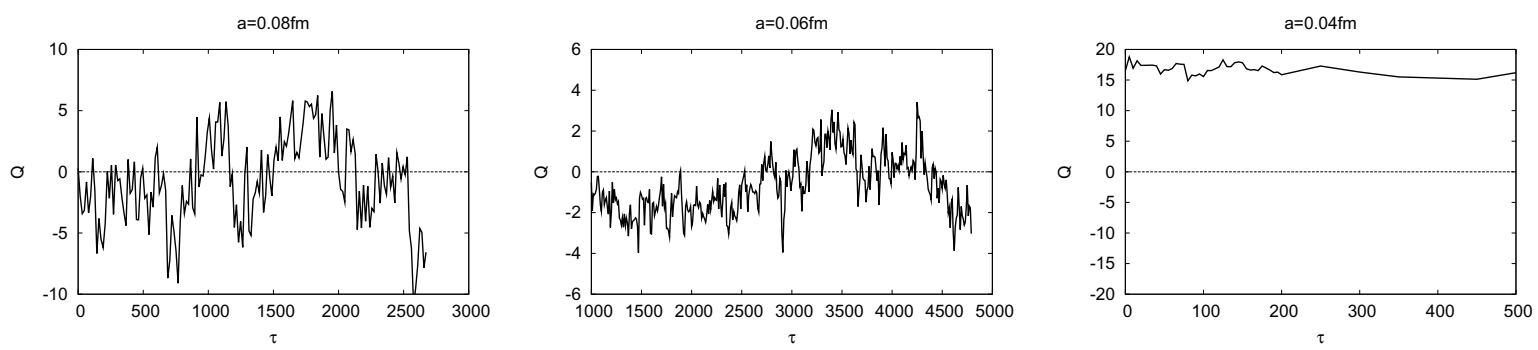

Figure 16: Histories of an estimate of the topological charge on runs with $N_{\mathrm{f}}=2$ [74].

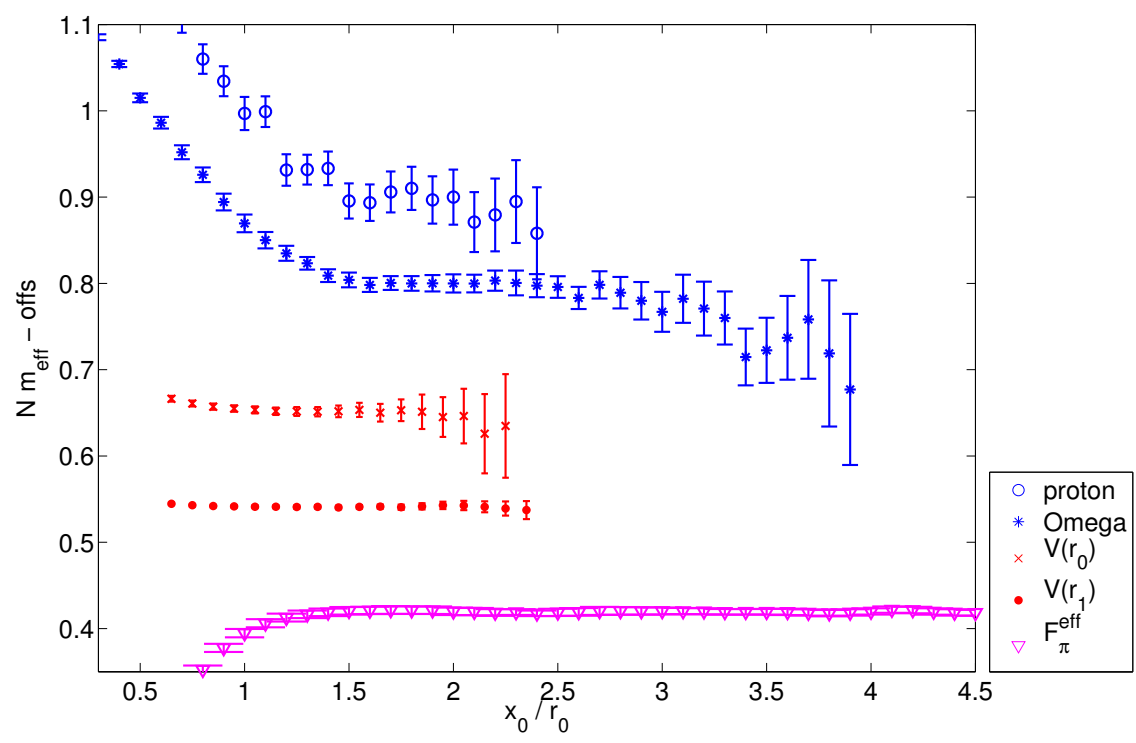

Figure 17: Effective masses for $m_{\mathrm{p}}$ [75], $m_{\Omega}$ [76], $V\left(\approx r_{0}\right), V\left(\approx r_{1}\right)$ [69] and $f_{\pi}$ [77] on CLS ensemble N6 (see [77]). All effective "masses" have been scaled such that the errors in the graph reflect directly the errors of the determined scales. They have been shifted vertically. Figure from [78].

off and finite volume effects. The latter would otherwise contaminate all quantities cited in $\mathrm{MeV}$ even if in lattice units they themselves are well controlled. Another point is that it is desirable to use a scale that is not very sensitive to the precise quark content and to the tuning of the masses of included sea quarks to their physical values. This is obviously only possible to a limited precision and in addition, for algorithmic reasons, the up/down quarks in simulations are most of the time heavier than in Nature and a chiral extrapolation is invoked. This step is theory guided by chiral perturbation theory, but it is clearly good to keep the general scale as independent of this as possible. In addition the effective theory with $N_{\mathrm{f}}<6$ is an approximation only and scale setting should be carried out in the sector of the theory that is robust against these presently still unavoidable small errors.
With this said, estimates for a number of quantities that are considered by the community are displayed in figure 17. The plot includes the effective masses of the proton and the $\Omega$ particle made of three strange quarks (still quenched for $N_{\mathrm{f}}=2$ ). We see the relatively large errors combined with short plateaus, where the situation for the $\Omega$ is somewhat superior. The static quark potential $V\left(r_{0}\right)$ is closely related to $r_{0}$ in (98) and $V\left(r_{1}\right)$ is a similar quantity where 1.65 is replaced by 1.00 . The lowest line with a very convincing plateau with small errors represents an effective matrix element corresponding to the decay constant $f_{\pi}$. A further bonus of such quantities is that they have been confirmed to be only weakly coupled to the slow modes responsible for topological freezing. Therefore a reliable error estimation is still possible down to the smallest lattice spacings $a \approx 0.05 \mathrm{fm}$ entering here. A small draw back asso- 
ciated with decay constants has to be mentioned too: to cite a physical value for them based on physical decays, separate experimental input for the relevant CKM matrix elements has to be used.

What has been said about $f_{\pi}$ is also true for the Kaon decay constant $f_{\mathrm{K}}$. It is our preferred scale setting quantity in this study, because of two more bonuses. The chiral extrapolation from larger pion masses in the range of around $500 \mathrm{MeV}$ to $270 \mathrm{MeV}$ down to the physical value is simpler for $f_{\mathrm{K}}$. In this case the partially quenched variant of chiral perturbation theory (pqChPT) is used. A purely technical point to prefer the $\mathrm{K}$ sector in our $N_{\mathrm{f}}=2$ simulations is that the strange quark mass can here be varied after the run without having to generate new configurations. Only a few extra inversions for the strange quark propagator are needed which is a relatively small effort.

\subsection{Scale setting for $N_{\mathrm{f}}=2$}

We start with a compilation of simulation data in tables 2 and 1 . The non-integer values for $L_{\max } / a$ derive from interpolations, see [69] for more details.

The main difficulty and source of a systematic error is the extrapolation to the proper quark masses, the "physical point". Once we decide to set the scale through $f_{\mathrm{K}}$, this point is naturally defined by

$$
R_{\mathrm{K}}=R_{\mathrm{K}}^{\text {phys }}, \quad R_{\pi}=R_{\pi}^{\text {phys }},
$$

where

$$
R_{\mathrm{K}}=\frac{m_{\mathrm{K}}^{2}}{f_{\mathrm{K}}^{2}}, \quad R_{\pi}=\frac{m_{\pi}^{2}}{f_{\mathrm{K}}^{2}},
$$

and $R_{\mathrm{K}}^{\mathrm{phys}}, R_{\pi}^{\mathrm{phys}}$ are the values of these ratios in Nature. In an attempt to minimize uncertainties, we take the physical masses and decay constants to be the ones in the isospin symmetric limit with QED effects removed as discussed in [80]. We use

$$
m_{\pi, \text { phys }}=134.8 \mathrm{MeV}, m_{\mathrm{K}, \text { phys }}=494.2 \mathrm{MeV} .
$$

One can then, for each lattice spacing, carry out two different strategies for extrapolating to the physical point.

In strategy 1 one keeps

$$
R_{\mathrm{K}} \equiv \frac{m_{\mathrm{K}}^{2}}{f_{\mathrm{K}}^{2}}=R_{\mathrm{K}}^{\text {phys }},
$$

fixed, as one varies the light (dynamical) quark mass. The condition defines a curve in the quark mass plane spanned by $\left(m_{\mathrm{d}}=\right) m_{\mathrm{u}}$ and $m_{\mathrm{s}}$. It is a very interesting one because along that curve $m_{\mathrm{K}}$ is constant up to terms of order $m_{\mathrm{u}}^{2}$. In the ChPT expansion the mass-dependence is then small and in particular the coefficient of the chiral $\log , m_{\pi}^{2} \log m_{\pi}^{2}$, is small. One expects that $f_{\mathrm{K}}$ can be extrapolated rather easily to the physical point along this curve.

The order $m_{\mathrm{u}}^{2}$ corrections are known in terms of one low energy constant, $\alpha_{4}$. One just has to implement our condition eq. (107), which expresses $m_{\mathrm{K}}$ in terms of $m_{\pi}$, in the formulae of [81].

The predicted form is

$$
\begin{aligned}
f_{\mathrm{K}}= & f_{\mathrm{K}, \text { phys }}\left[1+\bar{L}_{\mathrm{K}}\left(y_{1}, y_{\mathrm{K}}\right)\right. \\
& +\left(\alpha_{4}-\frac{1}{4}\right)\left(y_{1}-y_{\pi}\right)+\mathrm{O}\left(y^{2}\right), \\
\bar{L}_{\mathrm{K}}\left(y_{1}, y_{\mathrm{K}}\right)= & L_{\mathrm{K}}\left(y_{1}, y_{\mathrm{K}}\right)-L_{\mathrm{K}}\left(y_{\pi}, y_{\mathrm{K}}\right) \\
L_{\mathrm{K}}\left(y_{1}, y_{\mathrm{K}}\right)= & -\frac{1}{2} y_{1} \log \left(y_{1}\right) \\
& -\frac{1}{8} y_{1} \log \left(2 y_{\mathrm{K}} / y_{1}-1\right)
\end{aligned}
$$

The variables

$$
\begin{aligned}
y_{1} & =\frac{m_{\pi}^{2}}{8 \pi^{2} f_{\mathrm{K}}^{2}}, \\
y_{\pi} & =\frac{m_{\pi, \mathrm{phys}}^{2}}{8 \pi^{2} f_{\mathrm{K}, \text { phys }}^{2}}=0.00958, \\
y_{\mathrm{K}} & =\frac{m_{\mathrm{K}, \text { phys }}^{2}}{8 \pi^{2} f_{\mathrm{K}, \text { phys }}^{2}}=0.12875,
\end{aligned}
$$

are proportional to (averages of) quark masses up to quadratic terms. Because of eq. (107), we have $y_{3} \equiv$ $m_{\mathrm{K}}^{2} /\left[8 \pi^{2} f_{\mathrm{K}}^{2}\right]=2 y_{\mathrm{K}}-y_{1}+\mathrm{O}\left(y^{2}\right)$ and $y_{3}$ does not appear in eq. (108).

Another option is strategy 2, where one keeps the (PCAC) strange quark mass fixed and uses the expansion in just the up quark mass, i. e. SU(2) chiral perturbation theory adopted to this situation.

Figure 18 shows extrapolations with both strategies, which converge well at the physical point.

It remains to perform a continuum extrapolation of the dimensionless combination $f_{\mathrm{K}} L_{\max }$ with the help of interpolations of the integer $L_{\max } / a$ as a function of the bare coupling $g_{0}^{2}$. Little discretisation errors are seen in figure 19 which leads to the continuum limit

$$
f_{\mathrm{K}} L_{\max }=0.315(8)(2) \text {. }
$$

As in the previous section, the final results come from strategy 1 for the chiral extrapolation of $F_{\mathrm{K}}$. Strategy 2 is used to estimate the systematic uncertainty in the second parenthesis; it is small compared to the statistical errors. We then quote

$$
\Lambda_{\mathrm{SF}}^{(2)} / f_{\mathrm{K}}=0.84(6) \text {. }
$$




\begin{tabular}{ccccccc}
\hline$L / a$ & $\beta$ & $\kappa_{\text {sea }}$ & $a m$ & $\bar{g}^{2}(L)$ & $\delta\left[\bar{g}^{2}\right]$ & $L_{\max } / a$ \\
\hline 4 & 5.2000 & 0.134700 & $-0.03745(41)$ & $3.730(11)$ & & \\
4 & 5.2000 & 0.133780 & $-0.00086(35)$ & $3.797(11)$ & & \\
4 & 5.2000 & - & $\rightarrow 0$ & $3.798(11)$ & -0.686 & $5.11(3)(13)$ \\
6 & 5.2000 & 0.135600 & $-0.01322(26)$ & $4.810(32)$ & & \\
6 & 5.2000 & 0.135200 & $+0.00289(24)$ & $4.984(33)$ & & \\
6 & 5.2000 & - & $\rightarrow 0$ & $4.954(33)$ & +0.470 & $5.33(4)(11)$ \\
\hline 6 & 5.2638 & 0.135673 & $+0.00012(19)$ & $4.550(25)$ & +0.066 & $5.89(4)(2)$ \\
8 & 5.4689 & 0.136575 & $+0.00046(11)$ & $4.526(32)$ & +0.042 & $7.91(7)(1)$ \\
10 & 5.6190 & 0.136700 & $+0.00038(8)$ & $4.531(51)$ & +0.037 & $9.87(14)(2)$ \\
\hline \hline 12 & 5.7580 & 0.136623 & $+0.00067(7)$ & $4.501(91)$ & +0.017 & $11.94(31)(1)$ \\
16 & 5.9631 & 0.136422 & $-0.00096(4)$ & $4.40(10)$ & +0.084 & $16.40(50)(6)$ \\
\hline
\end{tabular}

Table 1: Values of $L_{\max } / a$ after correcting the simulated values $L / a$ to match the target $\bar{g}$ from [69]. The data at the two largest $\beta$-values are from [79]. The second error on the final result is the systematic one.

\begin{tabular}{ccccc}
\hline$\beta$ & $L_{\max } / a$ & $L_{\max } f_{\mathrm{K}}$ & $r_{0} / L_{\max }$ & $\bar{m}_{\text {strange }} / f_{\mathrm{K}}$ \\
\hline 5.2 & $5.367(82)$ & $0.318(6)(3)$ & $1.155(22)$ & $0.530(12)(6)$ \\
5.3 & $6.195(51)$ & $0.320(5)(4)$ & $1.169(15)$ & $0.577(11)(7)$ \\
5.5 & $8.280(80)$ & $0.316(4)(2)$ & $1.213(17)$ & $0.617(11)(5)$ \\
\hline cont. & & $0.315(8)(2)$ & $1.252(33)$ & $0.678(12)(5)$ \\
\hline
\end{tabular}

Table 2: Values of $L_{\max } / a, L_{\max } f_{\mathrm{K}}, r_{0} / L_{\max }$ and $\bar{m}_{\text {strange }} / f_{\mathrm{K}}$ together with the values extrapolated to the continuum limit. The running mass in the Schrödinger Functional scheme $\bar{m}_{\text {strange }}$ is given at the renormalization scale $L_{\max }$.

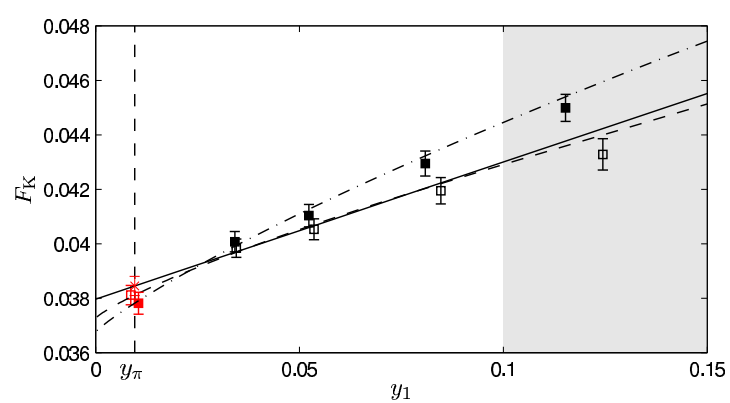

Figure 18: Physical point extrapolation of the kaon decay constant in lattice units. Open symbols and dashed lines correspond to strategy 1 , whereas filled symbols and dash-dotted lines represent strategy 2. Only data below $y_{1}=0.1$ enter the extrapolation. Figure from [69].

Now, as a result of our analysis, the error is dominated by the error on $\Lambda L_{\max }$. We translate to the $\overline{\mathrm{MS}}$ scheme using $\Lambda_{\mathrm{MS}}^{(2)}=2.382035(3) \Lambda_{\mathrm{SF}}^{(2)}[82,83]$ as well as to physical units

$$
\Lambda_{\mathrm{MS}}^{(2)}=310(20) \mathrm{MeV} \text {, }
$$

where

$$
f_{\mathrm{K}, \text { phys }}=155 \mathrm{MeV}
$$

enters.

As discussed previously with not all flavors of QCD treated dynamically there is a small ambiguity in the translation to $\mathrm{MeV}$. We therefore also give the result

$$
r_{0} \Lambda_{\overline{\mathrm{MS}}}^{(2)}=0.789(52),
$$

based on

$$
r_{0} / L_{\max }=1.252(33)
$$

in complete analogy to eq. (111). Our result eq. (115) is an unambiguous non-perturbative property of the twoflavor theory, sometimes called QCD-lite.

\subsubsection{Strange quark mass}

The RGI mass $M_{\mathrm{s}}$ is given in terms of the bare PCAC mass $m_{\text {strange }}$ by

$$
\begin{aligned}
M_{\mathrm{S}} & =\frac{M}{\bar{m}(L)} \bar{m}_{\text {strange }}(L) \\
& =\frac{M}{\bar{m}(L)} \frac{Z_{\mathrm{A}}}{Z_{\mathrm{P}}(L)} m_{\text {strange }},
\end{aligned}
$$

where $m_{\text {strange }}$ is the PCAC strange quark mass of strategy 2 . The continuum value of the universal first factor 


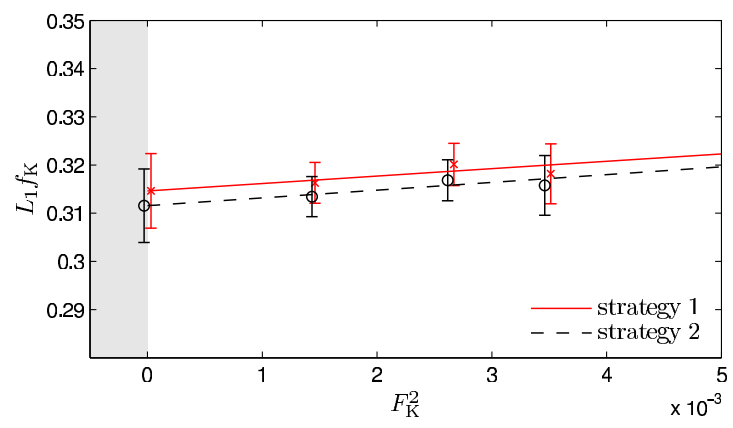

Figure 19: Continuum extrapolation of $L_{1}=L_{\max }$ in units of $f_{\mathrm{K}}$. Even though the data shows no cut-off effects, a linear extrapolation is used to account for uncertainties from $\mathrm{O}\left(a^{2}\right)$ effects hidden by the errors. The two strategies for the chiral extrapolation of $F_{\mathrm{K}}=a f_{\mathrm{K}}$ agree well within statistics.

$M / \bar{m}$ has been computed in Ref. [71] and discussed in section 7.3.

Expressing $M_{\mathrm{s}}$ in units of $f_{\mathrm{K}}$ we eliminate $Z_{\mathrm{A}}$ and get

$$
\begin{aligned}
& \frac{M_{\text {strange }}}{f_{\mathrm{K}, \text { phys }}}= \frac{M}{\bar{m}(L)} \times \frac{m_{\text {strange }}}{F_{\mathrm{K}, \text { phys }}^{\text {bare }}} \times \\
& \frac{1}{Z_{\mathrm{P}}(L)}\left[1+\left(\tilde{b}_{\mathrm{A}}-\tilde{b}_{\mathrm{P}}\right) a m_{\text {strange }}\right],
\end{aligned}
$$

with $F_{\mathrm{K} \text {,phys }}^{\text {bare }}=F_{\mathrm{K} \text {,phys }} / Z_{\mathrm{A}}$. The second factor is $\mathrm{O}(a)$ improved, if we neglect a tiny correction proportional to the sea quark mass, $\left(\bar{b}_{\mathrm{A}}-\bar{b}_{\mathrm{P}}\right) a m_{\text {sea }}$. Note that $\bar{b}_{\mathrm{A}}, \bar{b}_{\mathrm{P}}=$ $\mathrm{O}\left(g_{0}^{4}\right)$ are loop-suppressed and $a m_{\text {sea }}$ is very small.

Our final result is

$$
\begin{aligned}
\bar{m}_{\text {strange }} / f_{\mathrm{K}} & =0.678(12)(5), \\
M_{\mathrm{s}} / f_{\mathrm{K}} & =0.887(19)(7), \\
M_{\mathrm{S}} & =138(3)(1) \mathrm{MeV},
\end{aligned}
$$

where we use $M / \bar{m}=1.308(16)$ at the scale $L_{\max }$. For reference, we also give the numbers in the $\overline{\mathrm{MS}}$ scheme. This conversion is the only part of the computation in which we need to take recourse to perturbation theory, known in this case to four loops [84, 85, 86, 87], which differs from the two- and three-loop result by only a small amount. We use the same method as described in [71], but with the new value of $\Lambda_{\overline{\mathrm{MS}}}$ which leads us to $\bar{m}^{\overline{\mathrm{MS}}}(2 \mathrm{GeV}) / M=0.740(12)$ and

$$
\begin{aligned}
\bar{m}_{\mathrm{s}}^{\overline{\mathrm{MS}}}(2 \mathrm{GeV}) & =\frac{M_{\mathrm{s}}}{f_{\mathrm{K}}} \frac{\bar{m}^{\overline{\mathrm{MS}}}(2 \mathrm{GeV})}{M} f_{\mathrm{K}, \text { phys }} \\
& =102(3)(1) \mathrm{MeV} .
\end{aligned}
$$

\subsection{Scale setting for $N_{\mathrm{f}}=2+1$}

After the progress in simulation algorithms and the understanding of how to get around the topological freezing, CLS has started large-scale QCD simulations with a strange quark in addition to degenerate up and down quarks. The action $S_{\mathrm{LW} \text { :SWsf }}$ is used with nonperturbative $c_{\mathrm{SW}}$ [31]. The simulations started just about $1 \frac{1}{2}$ years ago, but have already reached a similar coverage of lattice spacings and pion masses as the $N_{\mathrm{f}}=$ 2 simulations carried out before. A summary of the presently available ensembles is found in table 3 from [88] where the details of the simulations are described.

With a dynamical strange quark, the choice of the curve in the quark mass plane that one chooses to approach the physical point (cf. Sect. 8.3) is much more important since each choice of the strange quark mass means a new simulation. Various considerations enter into a choice of a trajectory. Strategy 2 of Sect. 8.3 seems a natural one, but one has to keep the renormalized strange mass fixed and in the non-degenerate case, the renormalization of the quark mass contains a mixture of the flavor singlet and the flavor non-singlet mass terms, which renormalize differently. Similarly the $\mathrm{O}(a)$ terms become more complicated[89]. As a result of this it is technically simpler to keep the trace of the quark mass matrix constant as one changes the light quark mass[90]. This condition is similar but not the same as strategy 1 . The most important simplification is that up to a (supposedly small) $\mathrm{O}(a)$ term, the condition of a fixed trace of the renormalized mass matrix is equivalent to

$$
\sum_{f=1}^{3} \frac{1}{\kappa_{f}}=\text { const } .
$$

It can thus be followed without any tuning errors. A non-trivial point is of course to choose the right value of the trace, the one which leads to a trajectory through the physical point. Slightly wrong choices and subsequent corrections are unavoidable. We skip this issue here.

The scale setting will proceed in analogy to Sect. 8.3 through the decay constants $f_{\pi}, f_{\mathrm{K}}$. With open boundary conditions translation invariance in time is lost. Boundary-effects of correlation functions exist and have to be taken into account. The theoretical analysis of these effects is the same as with SF boundary conditions in a large volume [91]. Numerical aspects are presently being studied in detail $[92,88]$. A short summary is that the boundary conditions do not present an obstacle for the extraction of the hadronic matrix elements such as decay constants. In fact for some cases they may be advantageous compared to the conventional torus, where particles may propagate around the periodic time. With open boundary conditions such effects are avoided.

We show an example of an open boundary condi- 


\begin{tabular}{ccccllccc}
\hline id & $\beta$ & $N_{\mathrm{s}}$ & $N_{\mathrm{t}}$ & $\kappa_{u}$ & $\kappa_{s}$ & $m_{\pi}[\mathrm{MeV}]$ & $m_{K}[\mathrm{MeV}]$ & $m_{\pi} L$ \\
\hline $\mathrm{B} 105$ & 3.40 & 32 & 64 & 0.136970 & 0.13634079 & 280 & 460 & 3.9 \\
$\mathrm{H} 101$ & 3.40 & 32 & 96 & 0.13675962 & 0.13675962 & 420 & 420 & 5.8 \\
$\mathrm{H} 102$ & 3.40 & 32 & 96 & 0.136865 & 0.136549339 & 350 & 440 & 4.9 \\
$\mathrm{H} 105$ & 3.40 & 32 & 96 & 0.136970 & 0.13634079 & 280 & 460 & 3.9 \\
$\mathrm{C} 101$ & 3.40 & 48 & 96 & 0.137030 & 0.136222041 & 220 & 470 & 4.7 \\
$\mathrm{D} 100$ & 3.40 & 64 & 128 & 0.137090 & 0.136103607 & 130 & 480 & 3.7 \\
\hline $\mathrm{H} 200$ & 3.55 & 32 & 96 & 0.137000 & 0.137000 & 420 & 420 & 4.4 \\
$\mathrm{~N} 200$ & 3.55 & 48 & 128 & 0.137140 & 0.13672086 & 280 & 460 & 4.4 \\
$\mathrm{D} 200$ & 3.55 & 64 & 128 & 0.137200 & 0.136601748 & 200 & 480 & 4.2 \\
\hline $\mathrm{N} 300$ & 3.70 & 48 & 128 & 0.137000 & 0.137000 & 420 & 420 & 5.1 \\
$\mathrm{~N} 301$ & 3.70 & 48 & 128 & 0.137005 & 0.137005 & 410 & 410 & 4.9 \\
$\mathrm{~J} 303$ & 3.70 & 64 & 192 & 0.137123 & 0.1367546608 & 260 & 470 & 4.1 \\
\hline
\end{tabular}

Table 3: List of present CLS ensembles with up, down and strange sea quarks and the action of [31]. The numbers for $m_{\pi}$ and $m_{\mathrm{K}}$ are rounded and use $\sqrt{8 t_{0}}=0.4144 \mathrm{fm}$. The lattice spacings are roughly $a=0.086 \mathrm{fm}, 0.064 \mathrm{fm}$ and $0.05 \mathrm{fm}$ for $\beta=3.4,3.55$ and 3.7, respectively. Table from [88].

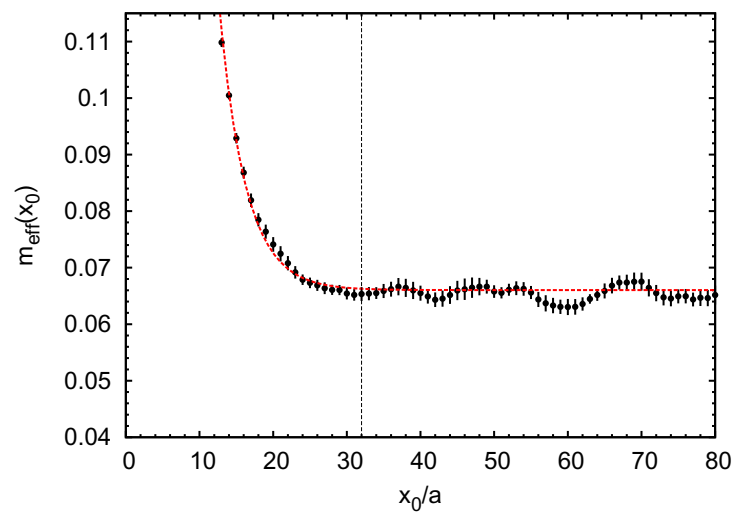

Figure 20: Effective pion mass of the D200 lattice (see table 3). Graph from from [88].

tion correlation function in figure 20, from [88]. Apart from the boundary effects the effective mass plot exhibts significant wiggles at large time, but these are of a purely statistical nature. Correlations between neighboring time-slices are very strong, but over larger distances also anticorrelations are present and in the end the fitted two-state curve is statistically compatible with the data points.

For the extraction of pseudo-scalar decay constants, just as for their mass, one has various possibilities to combine correlation functions. There numerical study revealed that the details do not matter too much and given a certain number of decorrelated configurations the statistical precision of these quantities is comparable to the one with periodic boundary condition ensembles
[92].

In summary, the $2+1$ simulations advance very fast and the scale setting is expected to be available rather soon.

\section{Outlook}

Let us first go back to figure 9. Here the present knowledge of the running of the SF coupling is summarized in an easily accessible way. For $N_{\mathrm{f}}=3$ the range of couplings is presently restricted. However, within this range the precision is already much better than for smaller flavor numbers. Still, the ALPHA collaboration is reducing the errors further. In parallel, work is now progressing on extending the range towards small energies. As explained earlier, in the lower energy region it becomes advantageous to use the flow coupling. Now that the reduction of cutoff effects of flow observables by Symanzik improvement is understood [40], we are ready to use it. The first preparation, the determination of the critical lines for different $L / a$ in the necessary region of larger $g_{0}$ as compared to figure 14 is already far advanced. The step scaling functions of $g_{\mathrm{GF}}$ will be evaluated soon.

As we have reported in the previous section, also the necessary large volume simulations are far advanced. Hence, we foresee to soon present the three flavor $\Lambda_{\overline{\mathrm{MS}}}^{(3)}$ with a precision that is at least comparable to the present FLAG average [6]. At this point, a perturbative relation of $\Lambda_{\overline{\mathrm{MS}}}^{(3)}$ to $\Lambda_{\overline{\mathrm{MS}}}^{(5)}$ can be used and one obtains an estimate of $\alpha_{\overline{\mathrm{MS}}}^{(5)}\left(M_{\mathrm{Z}}\right)$ with a precision of the non-lattice PDG average [93] or better. The additional uncertainty 
introduced by this step can roughly be divided into two pieces (for a precise description of the decoupling of heavy quarks on the non-perturbative level we refer to [5]). There are power-suppressed $\mathrm{O}\left(\left(M_{\text {charm }} / \Lambda_{\overline{\mathrm{MS}}}^{(4)}\right)^{-2}\right)$ terms due to the neglect of higher dimensional operators in the effective Lagrangian when we treat the low energy theory by just three flavor QCD. Such effects are entirely due to charm quark loops and are therefore suppressed by a factor of the number of colors in the large $N$ expansion on top of a perturbative suppression, i. e. by $\alpha / N$. In a (quite realistic, we would say) model it has recently been shown that indeed these power-suppressed terms are very small. We can safely neglect them within the envisaged accuracy. What remains is the matching of QCD with 3 flavors to QCD with 4 flavors. In terms of the $\Lambda$-parameters this is the relation

$$
\Lambda_{\overline{\mathrm{MS}}}^{(3)}=P_{3,4}\left(M_{\text {charm }} / \Lambda_{\overline{\mathrm{MS}}}^{(4)}\right) \Lambda_{\overline{\mathrm{MS}}}^{(4)},
$$

which has a perturbative expansion in terms of the coupling $\alpha(\mu)$ at the scale $\mu=M_{\text {charm. }}$. In the $\overline{\mathrm{MS}}$ scheme the relation is known to four loops and the resulting perturbative uncertainty looks very small [94], see [5] for the discussion of $P\left(M_{\text {charm }} / \Lambda_{\overline{\mathrm{MS}}}\right)$.

Nevertheless, perturbation theory at the scale $\mu=M_{\text {charm }}$ is worrying per se. Therefore the ALPHA collaboration also foresees a further step to carry out an adapted version of the full programme with four dynamical quark flavors. A first step is to bring Symanzik $\mathrm{O}(a)$ improvement under control with a heavy charm quark. We plan to carry out the steps at low and intermediate energy in a massive renormalization scheme [95]. Concerning improvement, this scheme does not need to be defined exactly with the charm mass at its physical value, but it is sufficient to have it fixed and close to it such that in an expansion in $a m_{\text {charm }}-a m_{\text {charm }}^{\text {phys }}$ one can safely neglect higher order terms. This is not an easy undertaking, but first steps are promising [95]. We can hence foresee to have in the near future a full four flavor non-perturbative determination of the $\Lambda$ parameter.

\section{Acknowledgements}

We would like to acknowledge the fruitful and pleasant collaboration with Mattia Bruno, Michele Della Morte, Patrick Fritzsch, Jochen Heitger, Roland Hoffmann, Andreas Jüttner, Francesco Knechtli, Tomasz Korzec, Björn Leder, Stefano Lottini, Marina Marinkovic, Harvey Meyer, Alberto Ramos, Juri Rolf, Stefan Schaefer, Stefan Sint, Hubert Simma, Felix Stollenwerk, Shinji Takeda, Fatih Tekin, Francesco Virotta, Ines Wetzorke and Oliver Witzel. The project is a completion of the ground-breaking work done in collaboration with Martin Lüscher and Peter Weisz whom we would like to thank very much for sharing their insights and support over the years. We thank Karl Jansen and Stefan Schaefer for reading and helping to improve an earlier version of this article.

We are grateful for the support of the Deutsche Forschungsgemeinschaft (DFG) in the SFB/TR 09 "Computational Particle Physics" and we have profited from the scientific exchange in the SFB.

Lastly, the results described here are also due to a lot of support for computational ressources. We gratefully acknowledge the Gauss Centre for Supercomputing (GCS) for providing computing time through the John von Neumann Institute for Computing (NIC) on the GCS share of the supercomputer JUQUEEN at Jülich Supercomputing Centre (JSC). GCS is the alliance of the three national supercomputing centres HLRS (Universität Stuttgart), JSC (Forschungszentrum Jülich), and LRZ (Bayerische Akademie der Wissenschaften), funded by the German Federal Ministry of Education and Research (BMBF) and the German State Ministries for Research of Baden-Württemberg (MWK), Bayern (StMWFK) and Nordrhein-Westfalen (MIWF). We acknowledge PRACE for awarding us access to resource JUQUEEN in Germany at Jülich. We thank the HLRN for time on the supercomputers Konrad and Gottfried and DESY for its support of the PAX cluster in Zeuthen.

\section{References}

[1] T. Appelquist, J. Carazzone, Infrared Singularities and Massive Fields, Phys.Rev. D11 (1975) 2856 doi:10.1103/PhysRevD.11.2856.

[2] S. Weinberg, Effective Gauge Theories, Phys.Lett. B91 (1980) 51. doi:10.1016/0370-2693(80)90660-7.

[3] S. Weinberg, Phenomenological Lagrangians, Physica A96 (1979) 327.

[4] W. Bernreuther, W. Wetzel, Decoupling of Heavy Quarks in the Minimal Subtraction Scheme, Nucl.Phys. B197 (1982) 228. doi:10.1016/0550-3213(82)90288-7.

[5] M. Bruno, J. Finkenrath, F. Knechtli, B. Leder, R. Sommer, On the effects of heavy sea quarks at low energies.arXiv: 1410.8374

[6] S. Aoki, Y. Aoki, C. Bernard, T. Blum, G. Colangelo, et al., Review of lattice results concerning low-energy particle physics, Eur.Phys.J. C74 (9) (2014) 2890. arXiv:1310.8555, doi:10.1140/epjc/s10052-014-2890-7.

[7] Z. Fodor, C. Hoelbling, Light Hadron Masses from Lattice QCD, Rev.Mod.Phys. 84 (2012) 449. arXiv:1203.4789, doi:10.1103/RevModPhys.84.449.

[8] K. Symanzik, Continuum Limit and Improved Action in Lattice Theories. 1. Principles and phi**4 Theory, Nucl.Phys. B226 (1983) 187. doi:10.1016/0550-3213(83)90468-6.

[9] K. Symanzik, Continuum Limit and Improved Action in Lattice Theories. 2. $\mathrm{O}(\mathrm{N})$ Nonlinear Sigma Model in Perturba- 
tion Theory, Nucl.Phys. B226 (1983) 205. doi:10.1016/05503213(83)90469-8.

[10] M. Lüscher, P. Weisz, On-Shell Improved Lattice Gauge Theories, Commun.Math.Phys. $97 \quad$ (1985) 59. doi:10.1007/BF01206178.

[11] M. Lüscher, Volume Dependence of the Energy Spectrum in Massive Quantum Field Theories. 1. Stable Particle States, Commun.Math.Phys. 104 (1986) 177. doi:10.1007/BF01211589.

[12] B. Chakraborty, C. Davies, G. Donald, R. Dowdall, B. Galloway, et al., High-precision quark masses and QCD coupling from $n_{f}=4$ lattice QCDarXiv:1408.4169.

[13] H. Casimir, On the Attraction Between Two Perfectly Conducting Plates, Indag.Math. 10 (1948) 261-263.

[14] M. Lüscher, P. Weisz, U. Wolff, A Numerical method to compute the running coupling in asymptotically free theories, Nucl.Phys. B359 (1991) 221-243. doi:10.1016/05503213(91)90298-C.

[15] K. G. Wilson, Confinement of Quarks, Phys.Rev. D10 (1974) 2445-2459. doi:10.1103/PhysRevD.10.2445.

[16] M. Lüscher, S. Sint, R. Sommer, P. Weisz, Chiral symmetry and $\mathrm{O}$ (a) improvement in lattice QCD, Nucl.Phys. B478 (1996) 365 400. arXiv:hep-lat/9605038, doi:10.1016/0550-3213(96)003781.

[17] B. Sheikholeslami, R. Wohlert, Improved Continuum Limit Lattice Action for QCD with Wilson Fermions, Nucl.Phys. B259 (1985) 572. doi:10.1016/0550-3213(85)90002-1.

[18] M. Lüscher, S. Sint, R. Sommer, P. Weisz, U. Wolff, Nonperturbative $\mathrm{O}(a)$ improvement of lattice QCD, Nucl. Phys. B491 (1997) 323-343. arXiv:hep-lat/9609035.

[19] M. Guagnelli, et al., Non-perturbative results for the coefficients $b_{m}$ and $b_{A}-b_{P}$ in $\mathrm{O}(a)$ improved lattice QCD, Nucl. Phys. B595 (2001) 44-62. arXiv:hep-lat/0009021.

[20] M. Lüscher, S. Sint, R. Sommer, H. Wittig, Nonperturbative determination of the axial current normalization constant in $\mathrm{O}(a)$ improved lattice QCD, Nucl. Phys. B491 (1997) 344-364. arXiv:hep-lat/9611015.

[21] K. Jansen, R. Sommer, $\mathrm{O}(a)$ improvement of lattice QCD with two flavors of Wilson quarks, Nucl. Phys. B530 (1998) 185 203. arXiv:hep-lat/9803017.

[22] M. Della Morte, R. Hoffmann, R. Sommer, Non-perturbative improvement of the axial current for dynamical Wilson fermions, JHEP 03 (2005) 029. arXiv:hep-lat/0503003.

[23] P. Fritzsch, J. Heitger, N. Tantalo, Non-perturbative improvement of quark mass renormalization in two-flavour lattice QCD, JHEP 1008 (2010) 074, arXiv:1004.3978.

[24] N. Yamada, et al., Non-perturbative o(a)-improvement of Wilson quark action in three-flavor QCD with plaquette gauge action, Phys. Rev. D71 (2005) 054505. arXiv:hep-lat/0406028.

[25] M. Lüscher, P. Weisz, $\mathrm{O}(a)$ improvement of the axial current in lattice QCD to one loop order of perturbation theory, Nucl. Phys. B479 (1996) 429-260. arXiv:hep-lat/9606016.

[26] S. Sint, P. Weisz, Further results on o( $a)$ improved lattice QCD to one loop order of perturbation theory, Nucl. Phys. B502 (1997) 251. arXiv:hep-lat/9704001.

[27] M. Lüscher, R. Narayanan, P. Weisz, U. Wolff, The Schrödinger Functional: A renormalizable Probe for non-Abelian Gauge Theories, Nucl. Phys. B384 (1992) 168. arXiv:hep-lat/9207009, doi:10.1016/0550-3213(92)90466-O.

[28] E. Seiler, Gauge Theories as a Problem of Constructive Quantum Field Theory and Statistical Mechanics, Lect.Notes Phys. 159 (1982) 1-192.

[29] R. Sommer, Non-perturbative QCD: Renormalization, O(a)improvement and matching to heavy quark effective theory, In Perspectives in Lattice QCD, World Scientific 2008arXiv:hep- lat/0611020

[30] S. Aoki, R. Frezzotti, P. Weisz, Computation of the improvement coefficient $\mathrm{c}(\mathrm{SW})$ to one loop with improved gluon actions, Nucl.Phys. B540 (1999) 501-519. arXiv:hep-lat/9808007, doi:10.1016/S0550-3213(98)00742-1.

[31] J. Bulava, S. Schaefer, Improvement of $N_{f}=3$ lattice QCD with Wilson fermions and tree-level improved gauge action, Nucl.Phys. B874 (2013) 188-197. arXiv:1304.7093, doi:10.1016/j.nuclphysb.2013.05.019.

[32] S. Sint, On the Schrodinger functional in QCD, Nucl.Phys. B421 (1994) 135-158. arXiv:hep-lat/9312079, doi:10.1016/0550-3213(94)90228-3.

[33] A. Chodos, R. Jaffe, K. Johnson, C. B. Thorn, Baryon Structure in the Bag Theory, Phys.Rev. D10 (1974) 2599 doi:10.1103/PhysRevD.10.2599.

[34] M. Lüscher, Construction of a Selfadjoint, Strictly Positive Transfer Matrix for Euclidean Lattice Gauge Theories, Commun.Math.Phys. 54 (1977) 283. doi:10.1007/BF01614090.

[35] M. Lüscher, Properties and uses of the Wilson flow in lattice QCD, JHEP 1008 (2010) 071. arXiv:1006.4518, doi:10.1007/JHEP08(2010)071, 10.1007/JHEP03(2014)092.

[36] P. Fritzsch, A. Ramos, The gradient flow coupling in the Schrdinger Functional, JHEP 1310 (2013) 008. arXiv:1301.4388, doi:10.1007/JHEP10(2013)008.

[37] A. Bode, P. Weisz, U. Wolff, Two loop computation of the Schrodinger functional in lattice QCD, Nucl.Phys. B576 (2000) 517-539. arXiv:hep-lat/9911018, doi:10.1016/S05503213(00)00187-5.

[38] M. Lüscher, P. Weisz, Perturbative analysis of the gradient flow in non-abelian gauge theories, JHEP 1102 (2011) 051 arXiv:1101.0963, doi:10.1007/JHEP02(2011)051.

[39] J. Zinn-Justin, D. Zwanziger, Ward Identities for the Stochastic Quantization of Gauge Fields, Nucl.Phys. B295 (1988) 297 doi:10.1016/0550-3213(88)90358-6.

[40] A. Ramos, S. Sint, On O $\left(a^{2}\right)$ effects in gradient flow observables, PoS LATTICE2014 (2014) 329. arXiv:1411.6706.

[41] Z. Fodor, K. Holland, J. Kuti, D. Nogradi, C. H. Wong, The Yang-Mills gradient flow in finite volume, JHEP 1211 (2012) 007. arXiv:1208.1051, doi:10.1007/JHEP11(2012)007.

[42] Lüscher, Martin, Computational Strategies in Lattice QCD (2010) 331-399arXiv: 1002.4232.

[43] S. Duane, A. Kennedy, B. Pendleton, D. Roweth, Hybrid Monte Carlo, Phys.Lett. B195 (1987) 216-222. doi:10.1016/03702693(87)91197-X.

[44] S. A. Gottlieb, W. Liu, D. Toussaint, R. L. Renken, R. L. Sugar, Hybrid Molecular Dynamics Algorithms for the Numerical Simulation of Quantum Chromodynamics, Phys. Rev. D35 (1987) 2531. doi:10.1103/PhysRevD.35.2531.

[45] W. H. Press, B. P. Flannery, S. A. Teukolsky, W. T. Vetterling, Numerical Recipes: The Art of Scientific Computing, Cambridge Univ. Press, Cambridge.

[46] Lüscher, Martin, Deflation acceleration of lattice QCD simulations, JHEP 0712 (2007) 011. arXiv:0710.5417, doi:10.1088/1126-6708/2007/12/011.

[47] Lüscher, Martin, Local coherence and deflation of the low quark modes in lattice QCD, JHEP 0707 (2007) 081 arXiv:0706.2298, doi:10.1088/1126-6708/2007/07/081.

[48] A. Frommer, K. Kahl, S. Krieg, B. Leder, M. Rottmann, Adaptive Aggregation Based Domain Decomposition Multigrid for the Lattice Wilson Dirac Operator, SIAM J.Sci.Comput. 36 (2014) A1581-A1608. arXiv:1303.1377, doi: $10.1137 / 130919507$.

[49] J. Sexton, D. Weingarten, Hamiltonian evolution for the hybrid Monte Carlo algorithm, Nucl.Phys. B380 (1992) 665-678 doi:10.1016/0550-3213(92)90263-B. 
[50] I. Omelyan, I. Mryglod, R. Folk, Symplectic analytically integrable decomposition algorithms: classification, derivation, and application to molecular dynamics, quantum and celestial mechanics simulations, Computer Physics Communications 151 (3) (2003) 272 - 314. doi:http://dx.doi.org/10.1016/S00104655(02)00754-3.

[51] M. Hasenbusch, Speeding up the hybrid Monte Carlo algorithm for dynamical fermions, Phys.Lett. B519 (2001) 177-182. arXiv:hep-lat/0107019, doi:10.1016/S0370-2693(01)01102-9.

[52] M. Hasenbusch, K. Jansen, Speeding up lattice QCD simulations with clover improved Wilson fermions, Nucl.Phys. B659 (2003) 299-320. arXiv:hep-lat/0211042, doi:10.1016/S05503213(03)00227-X.

[53] C. Urbach, K. Jansen, A. Shindler, U. Wenger, HMC algorithm with multiple time scale integration and mass preconditioning, Comput.Phys.Commun. 174 (2006) 87-98. arXiv:heplat/0506011, doi:10.1016/j.cpc.2005.08.006.

[54] M. Lüscher, S. Schaefer, Lattice QCD with open boundary conditions and twisted-mass reweighting, Comput.Phys.Commun. 184 (2013) 519-528. arXiv:1206.2809, doi:10.1016/j.cpc.2012.10.003.

[55] M. Clark, A. Kennedy, Accelerating dynamical fermion computations using the rational hybrid Monte Carlo (RHMC) algorithm with multiple pseudofermion fields, Phys.Rev.Lett. 98 (2007) 051601. arXiv:hep-lat/0608015, doi:10.1103/PhysRevLett.98.051601.

[56] U. Wolff, Monte Carlo errors with less errors, Comput.Phys.Commun. 156 (2004) 143-153. arXiv:heplat/0306017, doi:10.1016/S0010-4655(03)00467-3, 10.1016/j.cpc.2006.12.001

[57] Lüscher, M., Topology of Lattice Gauge Fields, Commun.Math.Phys. 85 (1982) 39. doi:10.1007/BF02029132.

[58] S. Schaefer, R. Sommer, F. Virotta, Critical slowing down and error analysis in lattice QCD simulations, Nucl.Phys. B845 (2011) 93-119. arXiv:1009.5228, doi:10.1016/j.nuclphysb.2010.11.020.

[59] P. Fritzsch, A. Ramos, F. Stollenwerk, Critical slowing down and the gradient flow coupling in the Schrdinger functional, PoS Lattice2013 (2014) 461. arXiv:1311.7304.

[60] M. Lüscher, S. Schaefer, Lattice QCD without topology barriers, JHEP 1107 (2011) 036. arXiv:1105.4749, doi:10.1007/JHEP07(2011)036.

[61] S. Schaefer, Status and challenges of simulations with dynamical fermions, PoS LATTICE2012 (2012) 001. arXiv:1211.5069.

[62] M. Della Morte, et al., Computation of the strong coupling in QCD with two dynamical flavors, Nucl.Phys. B713 (2005) 378-406. arXiv:hep-lat/0411025, doi:10.1016/j.nuclphysb.2005.02.013.

[63] S. Takeda, S. Aoki, M. Fukugita, K.-I. Ishikawa, N. Ishizuka, et al., A Scaling study of the step scaling function in SU(3) gauge theory with improved gauge actions, Phys.Rev. D70 (2004) 074510. arXiv:hep-lat/0408010, doi:10.1103/PhysRevD.70.074510.

[64] A. Bode, et al., First results on the running coupling in QCD with two massless flavors, Phys.Lett. B515 (2001) 49-56. arXiv:hep-lat/0105003, doi:10.1016/S0370-2693(01)00857-7.

[65] M. D. Brida, P. Fritzsch, T. Korzec, A. Ramos, S. Sint, et al., Towards a new determination of the QCD Lambda parameter from running couplings in the three-flavour theory, PoS LATTICE2014 (2014) 291. arXiv:1411.7648.

[66] M. Lüscher, R. Sommer, P. Weisz, U. Wolff, A Precise determination of the running coupling in the SU(3) Yang-Mills theory, Nucl.Phys. B413 (1994) 481-502. arXiv:hep-lat/9309005, doi:10.1016/0550-3213(94)90629-7.

[67] S. Capitani, M. Lüscher, R. Sommer, H. Wittig, Nonpertur- bative quark mass renormalization in quenched lattice QCD, Nucl.Phys. B544 (1999) 669-698. arXiv:hep-lat/9810063, doi:10.1016/S0550-3213(98)00857-8.

[68] J. Heitger, H. Simma, R. Sommer, U. Wolff, The Schrodinger functional coupling in quenched QCD at low- energies, Nucl.Phys.Proc.Suppl. 106 (2002) 859-861. arXiv:heplat/0110201, doi:10.1016/S0920-5632(01)01867-9.

[69] P. Fritzsch, F. Knechtli, B. Leder, M. Marinkovic, S. Schaefer et al., The strange quark mass and Lambda parameter of two flavor QCD, Nucl.Phys. B865 (2012) 397-429. arXiv:1205.5380, doi:10.1016/j.nuclphysb.2012.07.026.

[70] R. Sommer, A New way to set the energy scale in lattice gauge theories and its applications to the static force and alpha-s in SU(2) Yang-Mills theory, Nucl.Phys. B411 (1994) 839-854. arXiv:hep-lat/9310022, doi:10.1016/0550-3213(94)90473-1.

[71] M. Della Morte, et al., Non-perturbative quark mass renormalization in two-flavor QCD, Nucl.Phys. B729 (2005) 117-134. arXiv:hep-lat/0507035, doi:10.1016/j.nuclphysb.2005.09.028.

[72] Lüscher, Martin, Schwarz-preconditioned HMC algorithm for two-flavour lattice QCD, Comput.Phys.Commun 165 (2005) 199-220. arXiv:hep-lat/0409106, doi:10.1016/j.cpc.2004.10.004.

[73] M. Marinkovic, S. Schaefer, Comparison of the mass preconditioned HMC and the DD-HMC algorithm for two-flavour QCD, PoS LATTICE2010 (2010) 031. arXiv:1011.0911.

[74] S. Schaefer, R. Sommer, F. Virotta, Investigating the critical slowing down of QCD simulations, PoS LAT2009 (2009) 032. arXiv:0910.1465.

[75] B. Jäger, T. Rae, S. Capitani, M. Della Morte, D. Djukanovic, et al., A high-statistics study of the nucleon EM form factors, axial charge and quark momentum fraction, PoS LATTICE2013 (2014) 272. arXiv:1311.5804.

[76] S. Capitani, M. Della Morte, G. von Hippel, B. Knippschild, H. Wittig, Scale setting via the Omega baryon mass, PoS LATTICE2011 (2011) 145. arXiv:1110.6365.

[77] S. Lottini, Chiral behaviour of the pion decay constant in $N_{f}=2$ QCD, PoS LATTICE2013 (2014) 315. arXiv:1311.3081.

[78] R. Sommer, Scale setting in lattice QCD, PoS LATTICE2013 (2014) 015. arXiv:1401.3270.

[79] B. Blossier, et al., Parameters of Heavy Quark Effective Theory from $\mathrm{Nf}=2$ lattice QCD, JHEP 1209 (2012) 132. arXiv:1203.6516, doi:10.1007/JHEP09(2012)132.

[80] G. Colangelo, S. Dürr, A. Jüttner, L. Lellouch, H. Leutwyler, et al., Review of lattice results concerning low energy particle physicsarXiv:1011.4408.

[81] S. R. Sharpe, Enhanced chiral logarithms in partially quenched QCD, Phys.Rev. D56 (1997) 7052-7058. arXiv:heplat/9707018, doi:10.1103/PhysRevD.56.7052, 10.1103/PhysRevD.62.099901.

[82] M. Lüscher, R. Narayanan, P. Weisz, U. Wolff, The Schrödinger functional: A renormalizable probe for nonabelian gauge theories, Nucl. Phys. B384 (1992) 168-228. arXiv:hep-lat/9207009.

[83] S. Sint, R. Sommer, The running coupling from the QCD Schrödinger functional: A one loop analysis, Nucl. Phys. B465 (1996) 71-98. arXiv:hep-lat/9508012.

[84] T. van Ritbergen, J. A. M. Vermaseren, S. A. Larin, The four loop beta function in quantum chromodynamics, Phys. Lett. B400 (1997) 379-384. arXiv:hep-ph/9701390.

[85] K. G. Chetyrkin, Quark mass anomalous dimension to o (alpha-s**4), Phys. Lett. B404 (1997) 161-165. arXiv:hep$\mathrm{ph} / 9703278$.

[86] J. A. M. Vermaseren, S. A. Larin, T. van Ritbergen, The four loop quark mass anomalous dimension and the invariant quark mass, Phys. Lett. B405 (1997) 327-333. arXiv:hep-ph/9703284.

[87] M. Czakon, The Four-loop QCD beta-function and anoma- 
lous dimensions, Nucl.Phys. B710 (2005) 485-498. arXiv:hepph/0411261, doi:10.1016/j.nuclphysb.2005.01.012.

[88] M. Bruno, D. Djukanovic, G. P. Engel, A. Francis, G. Herdoiza, et al., Simulation of QCD with $N_{f}=2+1$ flavors of nonperturbatively improved Wilson fermionsarXiv:1411.3982.

[89] T. Bhattacharya, R. Gupta, W. Lee, S. R. Sharpe, J. M. $\mathrm{Wu}$, Improved bilinears in lattice QCD with non-degenerate quarks, Phys.Rev. D73 (2006) 034504. arXiv:hep-lat/0511014, doi:10.1103/PhysRevD.73.034504.

[90] W. Bietenholz, V. Bornyakov, N. Cundy, M. Gockeler, R. Horsley, et al., Tuning the strange quark mass in lattice simulations, Phys.Lett. B690 (2010) 436-441. arXiv:1003.1114, doi:10.1016/j.physletb.2010.05.067.

[91] M. Guagnelli, J. Heitger, R. Sommer, H. Wittig, Hadron masses and matrix elements from the QCD Schrodinger functional, Nucl.Phys. B560 (1999) 465-481. arXiv:hep-lat/9903040, doi:10.1016/S0550-3213(99)00466-6.

[92] M. Bruno, P. Korcyl, T. Korzec, S. Lottini, S. Schaefer, On the extraction of spectral quantities with open boundary conditions, PoS LATTICE2014 (2014) 089. arXiv:1411.5207.

[93] J. Beringer, et al., Review of Particle Physics (RPP), Phys.Rev. D86 (2012) 010001. doi:10.1103/PhysRevD.86.010001.

[94] K. Chetyrkin, J. H. Kuhn, C. Sturm, QCD decoupling at four loops, Nucl.Phys. B744 (2006) 121-135. arXiv:hepph/0512060, doi:10.1016/j.nuclphysb.2006.03.020.

[95] F. Stollenwerk, P. Fritzsch, R. Sommer, U. Wolff, Determination of $c_{\mathrm{SW}}$ in $N_{\mathrm{f}}=3+1$ Lattice QCD with massive Wilson fermions, PoS LATTICE2014 (2014) 293. 